\title{
Uniform independence for Dehn twist automorphisms of a free group
}

\author{
Edgar A. Bering IV
}

\begin{abstract}
McCarthy's Theorem for the mapping class group of a closed hyperbolic surface states that for any two mapping classes $\sigma, \tau \in \operatorname{Mod}(S)$ there is some power $N$ such that the group $\left\langle\sigma^{N}, \tau^{N}\right\rangle$ is either free of rank two or abelian, and gives a geometric criterion for the dichotomy. The analogous statement is false in linear groups, and unresolved for outer automorphisms of a free group. Several analogs are known for exponentially growing outer automorphisms satisfying various technical hypothesis. In this article we prove an analogous statement when $\sigma$ and $\tau$ are linearly growing outer automorphisms of $F_{r}$, and give a geometric criterion for the dichotomy. Further, Hamidi-Tehrani proved that for Dehn twists in the mapping class group this independence dichotomy is uniform: $N=4$ suffices. In a similar style, we obtain an $N$ that depends only on the rank of the free group.
\end{abstract}

\section{Introduction}

In the study of the analogy among linear groups, mapping class groups of surfaces, and outer automorphisms of free groups, the Tits alternative is a central achievement. McCarthy [26] and Ivanov [20] independently established a Tits alternative for mapping class groups. McCarthy's proof involves a more exact result for two generator subgroups (quoted below); an analogous statement is false for linear groups, the Heisenberg group is a counterexample. It is currently unknown whether $\operatorname{Out}\left(F_{r}\right)$ behaves like a linear group or a mapping class group in this setting, though there are many partial results, and this article adds another.

McCarthy's theorem for two-generator subgroups of the mapping class group of a surface $\Sigma$ can be viewed through the lens of a compatibility condition for geometric invariants associated to a pair of mapping classes. Recall that a mapping class $\sigma \in \operatorname{Mod}(\Sigma)$ is rotationless if every periodic homotopy class of curve is fixed. Associated to a rotationless mapping class is a decomposition of $\Sigma$ into invariant surfaces of negative Euler characteristic $\Sigma_{i}$ and annuli $A_{j}$, so that (up to isotopy) $\left.\sigma\right|_{\Sigma_{i}}$ is either identity or pseudo-Anosov, and $\left.\sigma\right|_{A_{j}}$ is some power of a Dehn twist about the core curve of $A_{j}$. The supporting lamination $\lambda$ of $\sigma$ is the union of the core curves of the non-trivial Dehn twist components (thought of as measured laminations with atomic measure equal to the absolute value of the twist power on the core curve) and the attracting measured laminations of the pseudo-Anosov components.

Theorem 1.1 McCarthy. Suppose $\sigma, \tau \in \operatorname{Mod}(\Sigma)$ are mapping classes of a closed hyperbolic surface $\Sigma$. Then there is an $N$ such that $\left\langle\sigma^{N}, \tau^{N}\right\rangle$ is either abelian or free of rank two. Moreover, $\left\langle\sigma^{N}, \tau^{N}\right\rangle \cong F_{2}$ exactly when $i(\lambda, \mu)>0$, where $\lambda$ and $\mu$ are the supporting measured laminations of rotationless powers of $\sigma$ and $\tau$ respectively.

2000 Mathematics Subject Classification 20E36 (primary), 20F65 (secondary).

This article is based upon the author's thesis submitted in partial fulfillment of the requirements for a $\mathrm{Ph}$. D. at the University of Illinois at Chicago 
Subsequent work of Hamidi-Tehrani [18, showed that when $\sigma$ and $\tau$ are Dehn twists, $N$ can be chosen independent of $\sigma, \tau$, and the surface. Building on this work and work of Fujiwara [14, Mangahas showed that if $\langle\sigma, \tau\rangle$ is not virtually abelian, then there is a $p$ depending only on the surface such that one of $\left\langle\sigma^{p}, \tau^{p}\right\rangle,\left\langle\sigma^{p}, \tau \sigma^{p} \tau^{-1}\right\rangle,\left\langle\sigma^{p}, \tau^{p} \sigma^{p} \tau^{-p}\right\rangle$, or $\left\langle\tau^{p}, \sigma^{p} \tau^{p} \sigma^{-p}\right\rangle$ is free of rank two, which implies that subgroups of $\operatorname{Mod}(S)$ which are not virtually abelian have uniform exponential growth, and the exponential growth rate depends only on $S$ [24]. Parallel results for $\operatorname{Out}\left(F_{r}\right)$ are unknown, and the main theorem of this article is a step towards them.

Using algebraic laminations an analogous result can be obtained for two generator subgroups of $\operatorname{Out}\left(F_{r}\right)$ when both generators are exponentially growing; this was first done by Bestvina, Feighn, and Handel [4] for pairs of fully irreducible outer automorphisms (with a novel proof using currents by Kapovich and Lustig [22]), and for exponentially growing outer automorphisms satisfying certain technical hypotheses by Taylor [31 and Ghosh [15. The techniques involved depend, in one way or another, on the existence of an attracting lamination for both generators. These approaches therefore do not apply to polynomially growing outer automorphisms, which have no laminations. Nevertheless, both Clay and Pettet [9] and Gultepe [17] prove that the subgroup of $\operatorname{Out}\left(F_{r}\right)$ generated by powers of "sufficiently independent" Dehn twists is free of rank two. Gultepe shows that any two Dehn twists satisfying a hypothesis on the geometry of their action on a certain complex generate a free group (without needing to pass to a power); while Clay and Pettet use tree theoretic methods to work with a larger family of twists, at the expense of a non-uniform power. (Unlike surface theory, there is no one-to-one correspondence between trees and laminations [27.)

In this article we make use of tree theoretic methods (one of which is a variation on Clay and Pettet's technique, itself an analog of Hamidi-Tehrani's methods). Two simplicial trees with $F_{r}$ action $A$ and $B$ are compatible if there is a tree $T$ with equivariant surjections $T \rightarrow A$ and $T \rightarrow B$ that collapse edges. Guirardel [16] introduced a geometric core of two trees and a notion of intersection number for these trees which measures compatibility. Guirardel shows that two simplicial trees are compatible if and only if $i(A, B)=0$ for this intersection number. Compatibility is exactly the notion needed to prove an analog of McCarthy's theorem for linearly growing outer automorphisms of $F_{r}$. Once more, certain periodic behavior poses a technical obstacle, but this can be avoided by passing to a uniform power.

Theorem 1.2 Main Theorem. Suppose $\sigma$ and $\tau$ are linearly growing outer automorphisms of $F_{r}$. For $N=\left(48 r^{2}-48 r+3\right)|G L(r, \mathbb{Z} / 3 \mathbb{Z})|$ the subgroup $\left\langle\sigma^{N}, \tau^{N}\right\rangle$ is either abelian or free of rank two. Moreover, the latter case holds exactly when $i(A, B)>0$ for the Bass-Serre trees $A$ and $B$ of efficient representatives of Dehn-twist powers of $\sigma$ and $\tau$.

We first introduce the relevant background facts regarding trees and their cores in Section 2 , and the necessary parts of the theory of $\operatorname{Out}\left(F_{r}\right)$ in Section 3 . The reader familiar with this theory can safely skim these sections for our notational conventions. To motivate the development of the tools needed in the proof of the main theorem, we examine a series of guiding examples, including the case of commuting twists and a setting similar to that considered by Clay and Pettet 9] in Section 4. The theme of the proof of the main theorem is to use the core: when it is a tree, it is a small tree mutually fixed by both automorphisms, and gives a commuting realization of the automorphisms. Should it fail to be a tree this failure will provide the geometric information needed to play ping-pong and find powers generating a free group. Sections 5 and 6 explore the geometric information obtained in detail, using the core to construct a simultaneous topological model of both tree actions. Finally, Section 7 completes the proof of the main theorem. 


\section{Trees and cores}

A simplicial tree is a contractible 1-dimensional cell complex. A tree can be given a metric by identifying each 1-cell with an interval $[a, b]$ (colloquially assigning each 1-cell a length), though the metric and CW-topologies will not agree in general. In this article we will always use the metric topology and if not otherwise specified we will use the metric given by assigning each 1-cell length one (this is often known as the path metric). A metric tree is uniquely geodesic, for any two points in $p, q$ the geodesic from $p$ to $q$ is the unique embedded arc joining $p$ and $q$. We will denote geodesics $[p, q]$ in this article, and use the convention that these geodesics are oriented; this treats $[q, p]$ as distinct from $[p, q]$ though they are the same set-wise. For an oriented geodesic $e, \bar{e}$ denotes its reverse.

Definition 2.1. A simplicial $F_{r}$-tree $T$ is an effective right action of the free group $F_{r}$ on a metric tree $T$ by isometries.

All trees in this article will be $F_{r}$-trees. We say an $F_{r}$-tree is minimal if there is no proper invariant subtree $T^{\prime} \subseteq T$; free when the action is free; irreducible when it is minimal, not a line, and the action does not fix an end; and small if the stabilizer of each edge is trivial or cyclic. Minimal small $F_{r}$-trees are irreducible [13]. A metric on an $F_{r}$-tree gives it a covolume, $\operatorname{covol}(T)$, the sum of the lengths of edges in the quotient $T / F_{r}$. Associated to an $F_{r}$-tree $T$ is a length function $\ell_{T}: F_{r} \rightarrow \mathbb{R}_{\geq 0}$ given by

$$
\ell_{T}(g)=\inf _{x \in T}\{d(x, x \cdot g)\} .
$$

Culler and Morgan [13] give a systematic treatment of (a generalization) of minimal $F_{r}$-trees via the associated length functions. For a fixed group element $g \in F_{r}$ the set

$$
C_{g}^{T}=\left\{x \in T \mid d(x, x \cdot g)=\ell_{T}(g)\right\}
$$

is always non-empty and is called the characteristic set of $g$. (When the tree $T$ is clear from context we suppress the superscript.) Elements with $\ell_{T}(g)>0$ are called hyperbolic and in this case $C_{g}^{T}$ is a line on which $g$ acts by translation by $\ell_{T}(g)$. This action gives $C_{g}^{T}$ a natural orientation, and rays contained in $C_{g}^{T}$ are referred to as either positive or negative according to this orientation (n.b. $C_{g^{-1}}^{T}$ has the reverse orientation, and gives the opposite classification to rays). There is a detailed relationship between length functions and axes elaborated on by Culler and Morgan, we need only a small piece here.

LEmma 2.2 [13]. Suppose $\ell(g h) \geq \ell(g)+\ell(h)$. Then there is a point $p \in C_{g}^{T} \cap C_{g h}^{T}$ such that $[p, p \cdot g] \subseteq C_{g h}^{T}$.

Proof. Culler and Morgan give a detailed construction of a fundamental domain for $C_{g h}^{T}$ in all cases. In the cases guaranteed by the hypothesis on the length function, this Culler-Morgan fundamental domain contains the desired arc.

Length functions provide a complete isometry invariant for irreducible $F_{r}$-trees, and embed the space of $F_{r}$-trees into $\mathbb{R}^{F_{r}}$ (one can restrict to conjugacy classes). The length function of any irreducible tree is non-zero, so this embedding projectivizes. The space of projective classes of free simplicial $F_{r}$-trees is projective Culler-Vogtmann outer space, $C V_{r}$; its closure $\overline{C V}_{r}$ in $\mathbb{P R}^{F_{r}}$ is compact [13]. Outer automorphisms act on length functions by pointwise composition, for $\phi \in \operatorname{Out}\left(F_{r}\right)$ and $\ell: F_{r} \rightarrow \mathbb{R}$ define $(\phi \ell)(g)=\ell(\phi(g))$, and this gives an action of $\operatorname{Out}\left(F_{r}\right)$ on $C V_{r}$ by homeomorphisms that extends to an action on $\overline{C V}_{r}$. 


\subsection{Very small trees and bounded cancellation}

The work of Cohen and Lustig combined with that of Bestvina and Feighn characterizes the $F_{r}$-trees representing projective classes in $\overline{C V}_{r}$ as the space of all very small real trees [7, 10]. (Real trees generalize simplicial trees, but are not needed for this article.)

Definition 2.3. A $F_{r}$-tree $T$ is very small if it is minimal, small, and has

(i) No obtrusive powers: for all $g \in F_{r} \backslash\{\operatorname{id}\}$ and $n$ such that $g^{n} \neq e, \operatorname{Fix}(g)=\operatorname{Fix}\left(g^{n}\right)$.

(ii) No tripod stabilizers: for all $a, b, c \in T$ such that the convex hull $H=H u l l(a, b, c)$ is not a point or arc, $\operatorname{Stab}(H)=\{\mathrm{id}\}$.

By virtue of their free simplicial approximability, many classical results about free groups have analogs for very small trees. One indispensable tool is Grayson and Thurston's bounded cancellation lemma, recorded by Cooper [12. Fix a basis for the free group $F_{r}$ and let $|\cdot|$ denote word length with respect to this basis. The classical bounded cancellation lemma states

Lemma 2.4 [12. Given an automorphism $f: F_{r} \rightarrow F_{r}$ there is a constant $C$ such that for all $w_{1}, w_{2} \in F_{r}$, if $\left|w_{1} w_{2}\right|=\left|w_{1}\right|+\left|w_{2}\right|$ then

$$
\left|f\left(w_{1} w_{2}\right)\right| \geq\left|f\left(w_{1}\right)\right|+\left|f\left(w_{2}\right)\right|-C .
$$

Let $T$ be the $F_{r}$-tree given by the Cayley graph of the fixed basis. An automorphism $f: F_{r} \rightarrow$ $F_{r}$ induces a Lipschitz equivariant map $\tilde{f}: T \rightarrow T ; \tilde{f}$ is the lift of some homotopy equivalence of a wedge of circles representing $f$. With the unit length metric, $|\cdot|$ gives the arc length for geodesics based at the identity. Lemma 2.4 implies that the geodesic from the identity to $w_{1} w_{2}$ is sent to the $\frac{C}{2}$ neighborhood of the geodesic from the identity to $f\left(w_{1} w_{2}\right)$. Since $\tilde{f}$ is equivariant, we conclude that for all finite geodesics $[p, q] \subseteq T, f([p, q])$ is in the $\frac{C}{2}$ neighborhood of the geodesic $[f(p), f(q)]$. This property generalizes to equivariant maps between trees.

DeFinition 2.5. An equivariant continuous map $f: S \rightarrow T$ between $F_{r}$-trees has bounded cancellation with constant $C$ if for all geodesics $[p, q] \subseteq S, f([p, q])$ is in the $C$ neighborhood of the $T$ geodesic $[f(p), f(q)]$.

In this form Bestvina, Feighn, and Handel give a bounded cancellation lemma for very small trees.

Lemma 2.6 [4, Lemma 3.1]. Suppose $T_{0}$ is a free simplicial $F_{r}$-tree and $T$ a very small $F_{r}$-tree, and $f: T_{0} \rightarrow T$ is an equivariant Lipschitz map. Then $f$ has a bounded cancellation constant $C(f)$ satisfying $C(f) \leq \operatorname{Lip}(f) \operatorname{covol}\left(T_{0}\right)$.

Their proof uses free simplicial approximation to bootstrap this result from Lemma 2.4. This lemma in turn implies a form of bounded cancellation for length functions of very small trees, reminiscent of the form of Lemma 2.4 (Kapovich and Lustig state a similar lemma, but with subtly different hypotheses [21]).

Lemma 2.7. Suppose $T$ is a very small $F_{r}$-tree and $\Lambda$ a basis for $F_{r}$. There is a constant $C(\Lambda, T)$ such that for all $g, h \in F_{r}$, if $|g h|_{\Lambda}=|g|_{\Lambda}+|h|_{\Lambda}$ and $g h$ is cyclically reduced with 


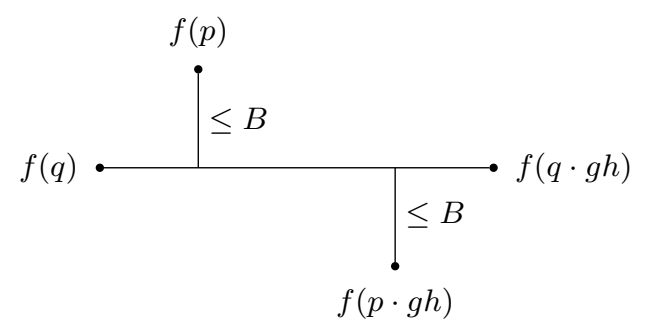

Figure 1. A convex hull in $T$.

respect to $\Lambda$, then

$$
\ell_{T}(g h) \geq \ell_{T}(g)+\ell_{T}(h)-C(\Lambda, T) .
$$

Further, $C(\Lambda, T) \leq 6 r \inf \operatorname{Lip}(f)$ where the infemum is taken over surjective Lipshitz maps $f: S_{\Lambda} \rightarrow T$ from the universal cover of a wedge of $r$ circles marked by the basis $S_{\Lambda}$.

Proof. Let $S_{\Lambda}$ be the universal cover of a wedge of $r$ circles with the circles marked by the basis $\Lambda$ where all edges have length one. Suppose $f: S_{\Lambda} \rightarrow T$ is an equivariant Lipschitz surjection. (Such maps always exist: pick zero cells $* \in S_{\Lambda}$ and $\star \in T$, define $f: S_{\Lambda}^{0} \rightarrow T$ on the zero skeleton by $f(* \cdot g)=\star \cdot g$ and extend linearly and equivariantly over edges. Since $S_{\Lambda}$ has finitely many edge orbits, this extension is Lipschitz. Moreover, $f$ is surjective since $T$ is minimal.) By Lemma 2.6 $f$ has bounded cancellation. Let $B$ be the bounded cancellation constant for $f$. Suppose $g, h \in F_{r}$ satisfy $|g h|_{\Lambda}=|g|_{\Lambda}+|h|_{\Lambda}$ and $g h$ is cyclically reduced. We will show that there is a constant $C$ depending on $\Lambda$ and $T$ such that for all $q \in S_{\Lambda}$,

$$
d(f(q), f(q \cdot g h)) \geq \ell_{T}(g)+\ell_{T}(h)-C .
$$

Since $f$ is equivariant and surjective, this implies the conclusion.

We will establish Equation 2.1 by showing that for any $q \in S_{\Lambda}$ there is a $p \in C_{g h}^{S_{\Lambda}}$ so that, for auxilliary constants $C^{\prime}$ and $C^{\prime \prime}$,

$$
d(f(q), f(q \cdot g h)) \geq d(f(p), f(p \cdot g h))-C^{\prime},
$$

and for all $p \in C_{g h}^{S_{\Lambda}}$,

$$
d(f(p), f(p \cdot g h)) \geq \ell_{T}(g)+\ell_{T}(h)-C^{\prime \prime} .
$$

Proof of Equation 2.2. Let $p$ be the point of $C_{g h}^{S_{\Lambda}}$ closest to $q$. The geodesic $[q, q \cdot g h]$ contains the points $p$ and $p \cdot g h$. Consider the convex hull in $T$ of $f(q), f(p), f(q \cdot g h)$, and $f(p \cdot g h)$ (Figure 1). Since the map $f$ has bounded cancellation, both $f(p)$ and $f(p \cdot g h)$ are in the $B$ neighborhood of the geodesic $[f(q), f(q \cdot g h)] \subset T$, and we have

$$
d(f(q), f(q \cdot g h)) \geq d(f(p), f(p \cdot g h))-2 B .
$$

Proof of Equation 2.3. Suppose now that $p \in C_{g h}^{S_{\Lambda}}$. We claim that it suffices to establish the inequality for the point $c$ that is the endpoint of the Culler-Morgan fundamental domain (Lemma 2.2) for the action of $g h$ on $C_{g h}^{S_{\Lambda}}$. To make this claim we first need to know the lemma applies. Since $g h$ is reduced and cyclically reduced, the word length equals the translation length of $g h$ on $S_{\Lambda}$, so that

$$
\ell_{S_{\Lambda}}(g h)=|g h|_{\Lambda}=|g|_{\Lambda}+|h|_{\Lambda} \geq \ell_{S_{\Lambda}}(g)+\ell_{S_{\Lambda}}(h) .
$$

Thus Lemma 2.2 applies and there is a $c \in C_{g h}^{S_{\Lambda}}$ such that $[c, c \cdot g] \subseteq C_{g h}^{S_{\Lambda}}$. 


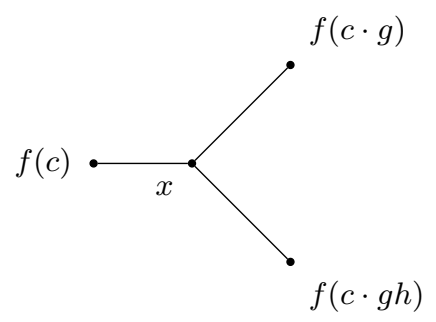

Figure 2. The triangle $f(c), f(c \cdot g), f(c \cdot g h)$ in $T$.

Continuing with the claim, without loss of generality we may assume that $c$ is between $p$ and $p \cdot g h$ on $C_{g h}^{S_{\Lambda}}$ by translating by $g h$ as needed. Consider the convex hull of $f(p), f(c), f(p$. $g h), f(c \cdot g h)$ in $T$. Let $x$ be the point on the geodesic $[f(p), f(c \cdot g h)]$ closest to $f(c)$ and $y$ the point closest to $f(p \cdot g h)$. Since $f$ has bounded cancellation, $d(f(c), x), d(f(p \cdot g h), y) \leq B$. We consider two cases, when $d(f(p), x)<d(f(p), y)$ and $d(f(p), y) \leq d(f(p), x)$. In both cases it will be important to note that, as $f$ is equivariant and the action is by isometry, $d(f(p), f(c))=$ $d(f(p \cdot g h), f(c \cdot g h))$.

In the first case, since $d(f(p), x)+d(x, f(c))=d(f(p \cdot g h), y)+d(y, f(c \cdot g h))$ and $x$ is on the geodesic $[f(c), f(c \cdot g h))]$ we have

$$
\begin{aligned}
d(f(p), f(p \cdot g h)) & =d(f(p), x)+d(x, y)+d(y, f(p \cdot g h)) \\
& =d(f(c \cdot g h), y)-d(f(c), x)+d(x, y)+2 d(y, f(p \cdot g h)) \\
& \geq d(f(c \cdot g h), f(c))-2 B .
\end{aligned}
$$

In the second case, the geodesic $[f(p), f(c)]$ contains $[y, x]$ (which may be a point), so we have $d(f(p), y)+d(y, x)+d(x, f(c))=d(f(p \cdot g h), y)+d(y, x)+d(x, f(c \cdot g h))$, and we calculate

$$
\begin{aligned}
d(f(p), f(p \cdot g h)) & =d(f(p), y)+d(y, f(p \cdot g h)) \\
& =d(f(c \cdot g h), x)-d(f(c), x)+2 d(y, f(p \cdot g h)) \\
& \geq d(f(c), f(c \cdot g h))-2 B .
\end{aligned}
$$

Hence, for any $p \in C_{g h}^{S_{\lambda}}$,

$$
d(f(p), f(p \cdot g h)) \geq d(f(c), f(c \cdot g h))-2 B
$$

and it remains to show that this is bounded below by translation lengths.

By construction, $c \cdot g$ is on the geodesic $[c, c \cdot g h]$. Consider the image of $c, c \cdot g$, and $c \cdot g h$ in $T$ and the geodesic triangle they span. Let $x \in T$ be the midpoint of this triangle (Figure 2). The bounded cancellation of $f$ implies that $d(x, f(c \cdot g)) \leq B$. We have

$$
\begin{aligned}
d(f(c), f(c \cdot g h)) & =d(f(c), f(c \cdot g))+d(f(c \cdot g), f(c \cdot g h))-2 d(x, f(c \cdot g)) \\
& \geq \ell_{T}(g)+\ell_{T}(h)-2 B
\end{aligned}
$$

establishing Equation 2.3 with $C^{\prime \prime}=4 B$.

Combining Equations 2.2 and 2.3. we have for all $q \in S_{\Lambda}$

$$
d(f(q), f(q \cdot g h)) \geq \ell_{T}(g)+\ell_{T}(h)-6 B
$$


and therefore,

$$
\ell_{T}(g h) \geq \ell_{T}(g)+\ell_{T}(h)-6 B .
$$

Finally, we note that this proof holds for all equivariant Lipschitz surjections $f: S_{\Lambda} \rightarrow T$, and by the previous bounded cancellation lemma $B \leq \operatorname{Lip}(f) \cdot \operatorname{covol}\left(S_{\Lambda}\right)=\operatorname{Lip}(f) \cdot r$. Taking an infimum over equivariant Lipschitz surjections $f: S_{\Lambda} \rightarrow T$ define $C(\Lambda, T)=6 r \inf \{\operatorname{Lip}(f)\}$. We conclude

$$
\ell_{T}(g h) \geq \ell_{T}(g)+\ell_{T}(h)-C(\Lambda, T)
$$

where the constant $C$ depends only on the basis and the very small tree $T$.

To apply this lemma effectively, it is useful to know when a good choice of basis exists or otherwise obtain control over the Lipshitz maps $f: S_{\lambda} \rightarrow T$. Lemma 3.13 is one example of such control.

\subsection{Bass and Serre's arboretum}

Bass and Serre 29] developed a detailed structure theory for groups acting on simplicial trees that relates the tree action to a generalization of an amalgamated product known as a graph of groups. Cohen and Lustig note that this theory applies equally well to metric trees [10. Below we recall key results of the theory and fix notation.

A graph $\Gamma$ is a collection of vertices $V(\Gamma)$, edges $E(\Gamma)$, initial and terminal vertex maps $o, t: E \rightarrow V$, and an involution $-: E \rightarrow E$, satisfying $\bar{e} \neq e$ and $o(\bar{e})=t(e)$. When there is a unique edge with $u=o(e)$ and $v=t(e)$ we will sometimes refer to $e$ as $(u, v)$. An assignment of lengths $d: E \rightarrow \mathbb{R}_{\geq 0}$ satisfying $d(e)=d(\bar{e})$ and $d(e)>0$ for all $e \in E$ is a metric on $\Gamma$. These edges are referred to as oriented edges, and a graph $\Gamma$ has a metric space realization by taking a point for each vertex, and attaching an interval of length $d(e)$ joining $o(e)$ and $t(e)$ for a set of representatives for the orbits of the involution ${ }^{-}$. An orientation of a graph $\Gamma$ is a set of orbit representatives for the involution. When working with graphs if not otherwise specifying a metric we will use the metric that assigns all edges length one.

A simplicial tree $T$ can be given a graph structure by taking branch points as vertices and adding a pair of edges $(p, q)=\overline{(q, p)}$ for each pair of vertices $p, q \in T^{(0)}$ such that $[p, q]$ is a 1simplex. Assigning lengths to one-simplices induces a metric on $T$ and a metric graph structure. The tree $T$ with this metric is the metric realization of this metric graph structure. When it is important to do so we will distinguish between a simplicial tree and a graph structure arising from a simplicial tree by calling the latter a graphical tree. A group $G$ acting on $T$ by simplicial isomorphism (isometry) naturally acts on this (metric) graph structure, and we say this action is without inversion if for all $e \in E(T)$ and $g \in G, e \cdot g \neq \bar{e}$. An action with inversion can be turned into an action without inversion by subdividing $T$.

Definition 2.8. A (metric) graph of groups is a pair $(G, \Gamma)$ where $\Gamma$ is a connected (metric) graph, and $G$ is an assignment of groups to the vertices and edges of $\Gamma$ satisfying $G_{e}=G_{\bar{e}}$, and injections $\iota_{e}: G_{e} \rightarrow G_{t(e)}$. We will often suppress the assignment $G$ and write $\Gamma_{e}, \Gamma_{v}$, etc.

The following applies to metric graphs of groups [10 equally well, but we make only light use of metric trees and can make do without belaboring the point.

The fundamental theorem of Bass-Serre theory gives an equivalence between actions on graphical trees and graphs of groups. Given a group $G$ acting on a graphical tree $T$, the quotient graph $\bar{T}$ has a graph of groups structure as follows. Pick a maximal subtree $S \subseteq \bar{T}$ and an orientation $Y$ of $\Gamma$. Define a section $j: \bar{T} \rightarrow T$ by first fixing a lift of $S$, and then for each $e \in Y \backslash E(S)$, define $j(e)$ so that $o(j(e))=j(o(e))$; also choose elements $\gamma_{e} \in G$ so 
that $t(j e)=\gamma_{e} j(t(e))$ for these edges. The assignment of $\gamma_{e}$ is extended to all of $E(\bar{T})$ by $\gamma_{\bar{e}}=\gamma_{e}^{-1}$ and $\gamma_{e}=1$ for $e \in E(S)$. Let $\chi$ be the indicator function for $E(\bar{T}) \backslash Y$. The graph of groups structure on $\bar{T}$ is given by $G_{v}=\operatorname{Stab}(j(v)), G_{e}=\operatorname{Stab}(j(e))$ and the inclusion maps by $\iota_{e}(a)=\gamma_{e}^{\chi(e)-1} a \gamma_{e}^{1-\chi(e)}$. Different choices of lift and maximal tree give isomorphic graphs of groups structures on the quotient, we say two graphs of groups are equivalent if they are different quotient labellings of the same tree.

Starting from a graph of groups $\Gamma$ there is an inverse operation, which recovers the group $G$ as the fundamental group of the graph of groups, and the tree $T$ that $G$ acts on so that the quotient is $\Gamma$. This is the Bass-Serre tree of $\Gamma$, the construction depends on a choice of maximal tree, and is unique up to equivariant isomorphism (isometry in the metric case). We will denote the quotient graph of groups by $\bar{T}$ and its tree $T$. When working with properties that are not conjugacy invariant the fundamental domain used will be specified.

The construction of the fundamental group of a graph of groups sits naturally in the context of the fundamental groupoid of a graph of groups, introduced by Higgins [19].

Definition 2.9. The fundamental groupoid $\pi_{1}(\Gamma)$ of a graph of groups $\Gamma$ is the groupoid with vertex set $V(\Gamma)$, generated by the path groupoid of $\Gamma$ and the groups $G_{v}$ subject to the following conditions. We require that for each $v \in V(\Gamma)$ the group $G_{v}$ is a sub-groupoid based at $v$ and that the group and groupoid structures agree. Further for all $e \in E(\Gamma)$ and $g \in G_{e}$, we have

$$
\bar{e} \iota_{\bar{e}}(g) e=\iota_{e}(g) .
$$

In particular this implies $\bar{e}$ and $e$ are inverse in $\pi_{1}(\Gamma)$.

By taking the vertex subgroup of $\pi_{1}(\Gamma)$ at a vertex $v$, we get the fundamental group $\pi_{1}(\Gamma, v)$. Changing basepoint results in an isomorphic group. The group $\pi_{1}(\Gamma, v)$ can also be described in terms of maximal trees. Fix a maximal tree $T$, and take the quotient of $\pi_{1}(\Gamma)$ by first identifying all vertices and then collapsing all edges of $T$. As explained by Higgins, it follows from standard results in groupoid theory that the result is isomorphic to $\pi_{1}(\Gamma, v)[19$.

Let $e=\left(e_{1}, e_{2}, \ldots, e_{n}\right)$ be a possibly empty edge path starting at $v$ and $g=\left(g_{0}, g_{1}, \ldots, g_{n}\right)$ a sequence of elements $g_{i} \in G_{t\left(e_{i}\right)}$ with $g_{0} \in G_{v}$. These data represent an arrow of $\pi_{1}(\Gamma)$ from $v$ to $t\left(e_{n}\right)$ by the groupoid product

$$
g_{0} e_{1} g_{1} \cdots e_{n} g_{n} .
$$

A non-identity element of $\pi_{1}(\Gamma)$ expressed this way is reduced if either $n=0$ and $g_{0} \neq \mathrm{id}$, or $n>0$ and for all $i$ such that $e_{i}=\bar{e}_{i+1}, g_{i} \notin G_{e_{i}}^{e_{i}}$. By fixing appropriate left transversals, a normal form for arrows of $\pi_{1}(\Gamma)$ is obtained. For each edge $e \in E(\Gamma)$, fix a left transversal $S_{e}$ of the image of $G_{e}$ in $G_{o(e)}$ containing the identity; by inductively applying the defining relations a reduced arrow is equivalent to a reduced arrow of the form

$$
s_{0} e_{0} s_{1} \cdots e_{n} h
$$

with each $s_{i} \in S_{e_{i}}$ and $h \in G_{t\left(e_{n}\right)}$. This representation is unique [19]. By specializing to $\pi_{1}(\Gamma, v)$ we obtain the Bass-Serre normal form for elements of the fundamental group based at $v$, with $h \in G_{v}$. This normal form depends on the choice of left-transversal, but the edges used do not.

For a conjugacy class $[g] \in \pi_{1}(\Gamma, v)$, a representative $g$ is cyclically reduced if it is reduced, $s_{0}=\mathrm{id}$, and $g$ has no sub-arrow $g^{\prime}$ based at $v$ such that $g=c g^{\prime} c^{-1}$ for $c \in \pi_{1}(\Gamma, v)$. In particular, if $o\left(e_{0}\right)=t\left(e_{0}\right)=v$, we have that if $\bar{e}_{n}=e_{0}$, then $h \notin \iota_{e_{n}}\left(G_{e_{n}}\right)$.

When $\pi_{1}(\Gamma, v)$ is free all vertex and edge groups are also free. A more refined normal form can be obtained by fixing an ordered basis $\Lambda$ for $\pi_{1}(\Gamma, v)$. Using the lexicographic order induced by $\Lambda$ and the Nielsen-Schreier theorem we obtain a unique minimal basis for each $G_{v}$. The induced 
order on the minimal bases of the $G_{v}$ specifies a unique minimal left Schreier transversal for the image of each $G_{e}$ with $t(e)=v$. Further, using the minimal right Schreier transversal $R_{e}$ of $G_{e}$ in $G_{t(e)}$ with respect to its preferred basis, we obtain a unique expression of the form

$$
x_{0} r_{0} e_{1} x_{1} r_{1} \cdots e_{n} x_{n} r_{n}
$$

where $x_{0} \in \iota_{e_{n}}\left(G_{e_{n}}\right)$, each $x_{i} \in \iota_{e_{i}}\left(G_{e_{i}}\right)$, and $r_{i} \in R_{e_{i}}$, and $x_{i} r_{i}$ reduced words with respect to the induced bases of the vertex groups. We call this the transverse Bass-Serre normal form with respect to $\Lambda$.

Definition 2.10. A graph of groups $\Gamma$ is minimal if for every connected proper subgraph $\Gamma^{\prime}$ and $v \in V\left(\Gamma^{\prime}\right)$ the induced map $\pi_{1}\left(\Gamma^{\prime}, v\right) \rightarrow \pi_{1}(\Gamma, v)$ is not surjective.

REMARK 2.11. This implies that if $v \in V(\Gamma)$ has valence one in a minimal graph of groups $\Gamma$, then $\iota_{e}\left(G_{e}\right)$ is not surjective, for the unique edge $e$ satisfying $v=t(e)$. As long as $\pi_{1}(\Gamma, v) \supsetneqq \mathbb{Z}$ or $D_{\infty}$, the resulting tree $T$ is then an irreducible $\pi_{1}(\Gamma, v)$-tree.

Proposition 2.12 [10, Proposition 9.2]. A graph of groups $\Gamma$ is minimal if and only if its Bass-Serre tree $T$ is a minimal $\pi_{1}(\Gamma, v)$ tree.

Proof. Cohen and Lustig leave this proof to the reader. We include it here. Suppose $\Gamma^{\prime} \subseteq \Gamma$ is a connected proper subgraph and $\pi_{1}\left(\Gamma^{\prime}, v\right) \rightarrow \pi_{1}(\Gamma, v)$ is surjective. Take a lift of $T^{\prime}$ (the tree of $\left.\Gamma^{\prime}\right)$ to $T$. This is a $\pi_{1}\left(\Gamma^{\prime}, v\right)$ invariant subtree by construction, and the action of $\pi_{1}(\Gamma, v)$ is induced by inclusion, so $T_{\Gamma^{\prime}}$ is a $\pi_{1}(\Gamma, v)$ invariant subtree, since the inclusion is surjective. Conversely, if $T^{\prime} \subseteq T$ is proper and $\pi_{1}(\Gamma, v)$ invariant, then $T^{\prime} / \pi_{1}(\Gamma, v)$ is a connected proper subgraph with graph of groups fundamental group $\pi_{1}(\Gamma, v)$, the induced inclusion map is an isomorphism.

To ensure that two minimal graphs of groups with equivariantly isometric Bass-Serre trees are isomorphic as graphs of groups a certain pathology must be excluded.

Definition 2.13. Let $\Gamma$ be a graph of groups. A valence two vertex $v \in V(\Gamma)$ with $v=$ $t\left(e_{1}\right)=t\left(e_{2}\right)$ is invisible if $\iota_{e_{1}}$ and $\iota_{e_{2}}$ are isomorphisms. If $\Gamma$ has no invisible vertices it is a visible graph of groups.

Invisible vertices are readily created by barycentric subdivision of edges and result in non-isomorphic simplicial structures on the Bass-Serre tree without changing the equivariant isometry class.

\subsection{Topological models}

Several authors give, in varying stages of development, an approach to building a topological model of a graph of groups $[1,8,28,32$. The treatment given by Scott and Wall is the popular reference 28, though Tretkoff's account includes a significantly more extensive discussion of the topological basis of normal forms 32. The definitions given by the various authors are equivalent in the cellular category, though the language is quite variable. This section will most closely follow Tretkoff's account. 
Definition 2.14. A graph of spaces $\mathcal{X}$ over a graph $\Gamma$ is a collection of cell complexes $\mathcal{X}$ indexed by the vertices and edges of $\Gamma$, such that $\mathcal{X}_{e}^{m}=\mathcal{X}_{\bar{e}}^{m}$, and cellular inclusions $\iota_{e}: \mathcal{X}_{e}^{m} \rightarrow$ $\mathcal{X}_{t(e)}$. The total space of $\mathcal{X}$, denoted $X$ is the quotient of the disjoint union

$$
\sqcup_{v \in V(\Gamma)} \mathcal{X}_{v} \sqcup_{e \in E(\Gamma)} \mathcal{X}_{e} \times[0,1]
$$

by the identifications

$$
\begin{aligned}
\mathcal{X}_{e}^{m} \times[0,1] & \rightarrow \mathcal{X}_{\bar{e}}^{m} \times[0,1] \quad(x, t) \mapsto(x, 1-t) \\
\mathcal{X}_{e}^{m} & \times 1 \rightarrow \mathcal{X}_{v} \quad(x, 1) \mapsto \iota_{e}(x)
\end{aligned}
$$

The total space $X$ of a graph of spaces over $\Gamma$ comes with a map $q: X \rightarrow \Gamma$ to the topological realization of $\Gamma$ by $q\left(\mathcal{X}_{v}\right)=v$ and $q\left(\mathcal{X}_{e}^{m} \times\{t\}\right)=e(t)$, the point of $e$ at coordinate $t$ realizing $e$ as the one-cell $[0,1]$. If $X$ is a cell complex with cellular map $q: X \rightarrow \Gamma$ such that the preimages of vertices and midpoints of edges gives a graph of spaces structure with $X$ as the total space, we say $q$ induces a graph of spaces structure on $X$. Note that the image of $\mathcal{X}_{e}^{m} \times[0,1]$ in $X$ is the double mapping cylinder on the two inclusion maps, we denote this image $\mathcal{X}_{e}$. (Indeed, some authors only require the maps be $\pi_{1}$ injective and construct the total space with the double mapping cylinder.) The spaces $\mathcal{X}_{e}^{m}$ naturally include into the total space $X$ via the map $\mathcal{X}_{e}^{m} \rightarrow \mathcal{X}_{e}^{m} \times\left\{\frac{1}{2}\right\}$, hence the superscript $m$ for midpoint.

By taking fundamental groups of the vertex and edge spaces of a graph of spaces we obtain an associated graph of groups assignment $G$ on $\Gamma$, and with $x \in \mathcal{X}_{v}, \pi_{1}(X, x) \cong \pi_{1}(\Gamma, v)$. This operation of course has an inverse, given a graph of groups $\Gamma$ a natural graph of spaces over $\Gamma$ can be constructed from $K\left(\Gamma_{v}, 1\right)$ and $K\left(\Gamma_{e}, 1\right)$ spaces. The group of deck transformations of the universal cover $\tilde{X}$ gives a definition of the fundamental group of $\Gamma$ that does not require a choice of basepoint or maximal tree.

Tretkoff gives a topological normal form for the homotopy class of a path relative to the endpoints in a graph of spaces, taking advantage of a classification of edges in the one skeleton. For a graph of spaces structure $\mathcal{X}$ with total space $X$, an edge in $X^{(1)}$ is $\mathcal{X}$-nodal if it lies in a vertex space, and $\mathcal{X}$-crossing otherwise. Tretkoff's form makes use of a fixed topological realization of the left transversals to ensure uniqueness, we need only the topological taxonomy of edges in the path, as formulated by Bestvina, Feighn, and Handel 6 .

Lemma 2.15 [6. Section 2.7; 32. Every path in a graph of spaces $X$ is homotopic relative to the endpoints to a path of the form (called normal form)

$$
v_{0} H_{1} v_{1} H_{2} \cdots H_{n} v_{n}
$$

where each $v_{i}$ is a (possibly trivial) tight edge path of $\mathcal{X}$-nodal edges, each $H_{i}$ is $\mathcal{X}$-crossing, and for all $1 \leq i \leq n-1, H_{i} v_{i} H_{i+1}$ is not homotopic relative to the endpoints to an $\mathcal{X}$-nodal edge path. Any two representatives of the homotopy class of a path in normal form have the same $n$. A similar statement holds for free homotopy classes of loops.

The proof of this lemma also illustrates that an edge path can be taken to normal form by iteratively erasing a pair of crossing edges; if $H_{i} v_{i} H_{i+1}$ is homotopic relative to the endpoints to a nodal edge path $v_{i}^{\prime}$ then the subpath $v_{i-1} H_{i} v_{i} H_{i+1} v_{i+1}$ is homotopic relative to endpoints to $v_{i-1} v_{i}^{\prime} v_{i+1}$ which can subsequently be tightened. Note that a path is in normal form if and only if every sub-path is. This should be compared to the normal form for arrows in the fundamental groupoid of a graph of groups, indeed one proof of the groupoid normal form is to prove this normal form and then apply the natural map from the fundamental groupoid of the total space $X$ to the fundamental groupoid of the graph of groups in question. 


\subsection{A core sampler}

Guirardel introduced the core of two real trees with group action to unify and generalize several intersection and compatibility phenomena in group theory.

Definition 2.16 [16]. The core of two simplicial $F_{r}$-trees $A$ and $B, \mathcal{C}(A, B)$ is the minimal non-empty closed subset of $A \times B$ with convex fibers invariant under the diagonal action of $G$. The augmented core $\widehat{\mathcal{C}}(A, B) \supseteq \mathcal{C}(A, B)$ is the minimal closed connected subset of $A \times B$ with convex fibers invariant under the diagonal action.

Remark 2.17. If $A$ and $B$ have minimal subtrees $A^{\prime}$ and $B^{\prime}$ then the core must be contained in $A^{\prime} \times B^{\prime}$.

Guirardel works in the much more general setting of group actions on real trees, but in this article we do not need to leave the cellular category; Guirardel shows if $A$ and $B$ are simplicial $G$-trees then $\mathcal{C}(A, B)$ is a square subcomplex of $A \times B$ [16, Proposition 2.6]. Further, for irreducible trees, the core is always non-empty, though it is not always connected.

The diagonal action of $F_{r}$ on $\mathcal{C}(A, B)$ induces a notion of covolume, while this notion is not well behaved in general, in the simplicial setting $\operatorname{covol}(\mathcal{C})$ is the total metric area of $\mathcal{C} / F_{r}$ (the number of squares when all edges of $A$ and $B$ have length one). Without a condition on the edge stabilizers of $A$ and $B$ this may be infinite, but we are concerned with the other extreme.

Definition 2.18. The intersection number of two simplicial $F_{r}$-trees $A$ and $B$ is

$$
i(A, B)=\operatorname{covol}(\mathcal{C}(A, B)) .
$$

For simplicial $F_{r}$-trees, the intersection number quantifies the (non)-existence of a common refining tree. Given two simplicial $F_{r}$ trees $A$ and $B$, we say that $T$ is a common refinement of $A$ and $B$ if there are equivariant surjections $f_{A}: T \rightarrow A$ and $f_{B}: T \rightarrow B$ that preserve alignment, the image of every geodesic $[p, q]$ is $\left[f_{S}(p), f_{S}(q)\right]$ with $S$ either $A$ or $B$. These maps arise from equivariantly collapsing edges.

Theorem 2.19 [16, Theorem 6.1]. Simplicial $F_{r}$-trees $A$ and $B$ have a common refinement if and only if $i(A, B)=0$. In this case $\widehat{\mathcal{C}}(A, B)$ is a common refinement.

In a previous paper [3] we give some equivalent characterizations of compatibility for irreducible $F_{r}$-trees that are useful for explicit computations (one of these generalizes a criterion of Behrstock, Bestvina, and Clay [2]). Let $e \subset T$ be an oriented edge in a simplicial $F_{r}$-tree. Let $\delta_{e}^{+}$be the connected component of $T \backslash e^{\circ}$ containing $t(e)$. The asymptotic horizon of $e$ is the set of group elements

$$
\llbracket e \rrbracket=\left\{g \in F_{r} \mid C_{g}^{T} \cap \delta_{e}^{+} \text {is a positive ray }\right\}
$$

Lemma 2.20 [3]. Suppose $A$ and $B$ are irreducible simplicial $F_{r}$-trees. The following are equivalent.

(i) $A$ and $B$ are not compatible.

(ii) There are edges $a \in E(A)$ and $b \in E(B)$ such that the four sets

$$
\llbracket a \rrbracket \cap \llbracket b \rrbracket, \llbracket \bar{a} \rrbracket \cap \llbracket b \rrbracket, \llbracket a \rrbracket \cap \llbracket \bar{b} \rrbracket, \llbracket \bar{a} \rrbracket \cap \llbracket \bar{b} \rrbracket
$$


are all non-empty.

(iii) There are group elements $g, h \in F_{r}$ such that

$$
\begin{aligned}
& \ell_{A}(g h)=\ell_{A}\left(g h^{-1}\right)>\ell_{A}(g)+\ell_{A}(h) \quad \text { and } \quad \ell_{B}(g h) \neq \ell_{B}\left(g h^{-1}\right) \\
& \text { or } \\
& \ell_{B}(g h)=\ell_{B}\left(g h^{-1}\right)>\ell_{B}(g)+\ell_{B}(h) \quad \text { and } \quad \ell_{A}(g h) \neq \ell_{A}\left(g h^{-1}\right) .
\end{aligned}
$$

The third condition is called incompatible combinatorics because of its implications about the combinatorial arrangement of axes and $A$ and $B$.

\subsection{The Bass-Serre case}

While not all useful stabilizer restrictions are retained by the core of compatible trees, when $A$ and $B$ are compatible Bass-Serre trees for graph of groups decompositions of $G$ the structure theory of the core permits a very explicit description of the augmented core.

Lemma 2.21. Suppose $\bar{A}$ and $\bar{B}$ are minimal visible graphs of groups with fundamental group $G ¥ \mathbb{Z}$ or $\mathbb{Z} / 2 \mathbb{Z} * \mathbb{Z} / 2 \mathbb{Z}$, and compatible Bass-Serre trees $A$ and $B$. The augmented core $\widehat{\mathcal{C}}(A, B)$ is then then the Bass-Serre tree for a graph of groups $\Gamma$ with fundamental group $G$, and the edge groups of $\Gamma$ are in the set of conjugacy classes of the edge groups of $\bar{A}$ and $\bar{B}$. Moreover, $\bar{A}$ and $\bar{B}$ are equivalent to graphs of groups $\bar{A}^{\prime}$ and $\bar{B}^{\prime}$ so that

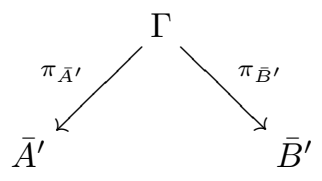

where $\pi_{\bar{A}^{\prime}}$ and $\pi_{\bar{B}^{\prime}}$ are quotient maps that collapse edges.

Proof. Guirardel proves that in this case the core is a common refinement and so $\widehat{\mathcal{C}}(A, B)$ is a simplicial $F_{r}$-tree (Theorem 2.19). Moreover, by the convexity of the fibers of the projection maps, the edges of $\widehat{\mathcal{C}}$ are of three forms

$$
\left\{v_{A}\right\} \times e_{B}, e_{A} \times\left\{v_{B}\right\}, \text { or } \Delta \subseteq e_{A} \times e_{B} .
$$

where $v_{T}$ and $e_{T}$ are vertices and edges in the trees $A$ and $B$. Further, using the equivariant projections from the core $\pi_{A}$ and $\pi_{B}$, we calculate stabilizers for each edge, $e \in \widehat{\mathcal{C}}(A, B)$

$$
\operatorname{Stab}_{\widehat{\mathcal{C}}(A, B)}(e)=\operatorname{Stab}_{A}\left(\pi_{A}(e)\right) \cap \operatorname{Stab}_{B}\left(\pi_{B}(e)\right) .
$$

Suppose $\pi_{A}(e)=a \in E(A)$. We claim

$$
\operatorname{Stab}_{\widehat{\mathcal{C}}(A, B)}(e)=\operatorname{Stab}_{A}(a) .
$$

Indeed, suppose there is some $g \in \operatorname{Stab}_{A}(a)$ but not in $\operatorname{Stab}_{B}\left(\pi_{B}(e)\right)$. Let $p \in a$ be the midpoint and let $q \in \pi_{B}(e)$ be any point. The point $(p, q)$ is in the interior of $e$, and since $g$ is not in the stabilizer, $(p \cdot g, q \cdot g)=(p, q \cdot g)$ is disjoint from $e$. Both $(p, q),(p, g \cdot q) \in \pi_{A}^{-1}(p)$, which is convex. However, the path in $\widehat{\mathcal{C}}(A, B)$ must pass through $o(e)$ or $t(e)$, neither of which is in $\pi_{A}^{-1}(p)$, a contradiction. Symmetrically, if $\pi_{B}(e)=b \in E(B)$ we find

$$
\operatorname{Stab}_{\widehat{\mathcal{C}}(A, B)}(e)=\operatorname{Stab}_{B}(b) .
$$


The remainder of the lemma is then immediate from standard facts in Bass-Serre theory, with $\widehat{\mathcal{C}}(A, B)$ the Bass-Serre tree of the desired graph of groups $\Gamma$. The graphs of groups $\bar{A}^{\prime}$ and $\bar{B}^{\prime}$ come from choosing a maximal tree and lift in $\Gamma$ and $\widehat{\mathcal{C}}(A, B)$, and projecting.

REMARK 2.22. This characterizes the edge groups of compatible graphs of groups: An edge group $\bar{A}_{e}$ is either conjugate to some $\bar{B}_{e}$ or contained within a conjugate of some $\bar{B}_{v}$, and vise-versa.

\section{Outer automorphisms}

By definition, the outer automorphism group $\operatorname{Out}\left(F_{r}\right)=\operatorname{Aut}\left(F_{r}\right) / \operatorname{Inn}\left(F_{r}\right)$ of a free group $F_{r}$ is the automorphism group modulo the inner automorphisms. We briefly review various topological perspectives on elements of $\operatorname{Out}\left(F_{r}\right)$, the classification by growth, and some details about representatives of outer automorphisms of linear growth.

\subsection{Topological representatives and growth}

Let $\Gamma$ be the realization of a graph with $\pi_{1}(\Gamma, v)=F_{r}$. An immersed path $\gamma:[0,1] \rightarrow \Gamma$ is tight if any lift $\tilde{\gamma}:[0,1] \rightarrow \tilde{\Gamma}$ is an embedding. Since $\tilde{\Gamma}$ is a tree, it is immediate that every immersed path is homotopic relative to the endpoints to a unique tight path, called its tightening. Given a path $\gamma$ we denote the tightening $[\gamma]$. Similarly, a closed loop is tight if it is tight for every choice of basepoint, and is freely homotopic to a unique tightening (a fundamental domain for the action of $\gamma_{*} \in \pi_{1}(\Gamma)$ on the universal cover $\tilde{\Gamma}$, with basepoint chosen on the axis of $\gamma_{*}$ ), the tightening of a loop $\gamma$ is denoted $[[\gamma]]$. Two paths $\gamma$ and $\delta$ are composable if the end of $\gamma$ equals the start of $\delta$, and their composition is denoted $\gamma \delta$; if $\gamma$ is a based loop $\gamma^{-1}$ denotes its reverse and $\gamma^{m}$ its $m$-fold concatenation for $m \in \mathbb{Z}$ (when $m=0$ this is a constant path at the basepoint of $\gamma$ ). A loop $\gamma$ is primitive if there is no $\gamma^{\prime}$ such that $[\gamma]=\left[\gamma^{\prime m}\right]$ for some $m>1$. We will assume from here on that all paths have endpoints at the vertices of $\Gamma$.

Given an outer automorphism $\sigma \in \operatorname{Out}\left(F_{r}\right)$, we can realize $\sigma$ as a homotopy equivalence $\hat{\sigma}: \Gamma \rightarrow \Gamma$. Such a realization is referred to as a topological representative; particularly nice topological representatives are indispensable in the analysis of outer automorphisms.

The growth of an outer automorphism is measured in terms of a topological representative. We say $\sigma$ is exponentially growing if there is some loop $\gamma \subseteq \Gamma$ such that $\ell_{\Gamma}\left(\left[\left[\hat{\sigma}^{n}(\gamma)\right]\right]\right)$ is bounded below by an exponential function, and that $\sigma$ is polynomially growing if there is some $d$ such that $\ell_{\Gamma}\left(\left[\left[\hat{\sigma}^{n}(\gamma)\right]\right]\right) \in O\left(n^{d}\right)$ for all loops $\gamma \subseteq \Gamma$. This classification does not depend on the choice of topological representative, as demonstrated by Bestvina, Feighn, and Handel [5]; the choice does matter for the details of the exponent in the exponentially growing case, however we are not concerned with exponentially growing outer automorphisms in this article.

Polynomially growing outer automorphisms can exhibit a certain amount of finite-order periodic behavior which results in significant technical headaches. These phenomena can be removed by passing to a uniform power. A polynomially growing outer automorphism $\sigma$ is unipotent if the induced action on the first homology $H_{1}\left(F_{r}, \mathbb{Z}\right)$ is a unipotent matrix. Bestvina, Feighn, and Handel proved that any polynomially growing outer automorphism that acts trivially on $H_{1}\left(F_{r}, \mathbb{Z} / 3 \mathbb{Z}\right)$ is unipotent [6. Proposition 3.5], so all polynomially growing outer automorphisms have a unipotent power. 


\subsection{Upper triangular representatives and the Kolchin theorem}

Unipotent polynomially growing outer automorphisms have particularly nice topological representatives. A homotopy equivalence $\hat{\sigma}: \Gamma \rightarrow \Gamma$ is filtered if there is a filtration $\emptyset=\Gamma_{0} \subsetneq$ $\Gamma_{1} \subsetneq \cdots \subsetneq \Gamma_{k}=\Gamma$ preserved by $\hat{\sigma}$.

Definition 3.1. A filtered homotopy equivalence $\hat{\sigma}$ is upper triangular if

(i) $\hat{\sigma}$ fixes the vertices of $\Gamma$,

(ii) Each stratum of the filtration $\Gamma_{i} \backslash \Gamma_{i-1}=E_{i}$ is a single topological edge,

(iii) Each edge $E_{i}$ has a preferred orientation and with this orientation there is a tight closed path $u_{i} \subseteq \Gamma_{i-1}$ based at $t\left(E_{i}\right)$ so that $\hat{\sigma}\left(E_{i}\right)=E_{i} u_{i}$.

The path $u_{i}$ is called the suffix associated to $u_{i}$, and when working with an upper triangular homotopy equivalences we will always refer to edges of the filtered graph with the preferred orientation. Just as paths have tightenings, if $\hat{\sigma}$ is a filtered homotopy equivalence that satisfies the above definition except that some $u_{i}$ is not tight, $\hat{\sigma}$ is homotopic to an upper triangular homotpy equivalence, also called its tightening. A filtration assigns to each edge a height, the integer $i$ such that $E \in \Gamma_{i} \backslash \Gamma_{i-1}$, and by taking a maximum this definition extends to tight edge paths. An upper-triangular homotopy equivalence preserves the height of each edge path.

Every upper triangular homotopy equivalence of a fixed filtered graph evidently induces a unipotent polynomially growing outer automorphism, and using relative train tracks Bestvina, Feighn, and Handel show the converse, every unipotent polynomially growing outer automorphism has an upper triangular representative [5, Theorem 5.1.8]. Moreover, for a given filtered graph $\Gamma$ the upper-triangular homotopy equivalences taken up to homotopy relative to the vertices form a group under composition. The suffixes for the inverse are defined inductively up the filtration by $\hat{\sigma}^{-1}\left(E_{i}\right)=E_{i} v_{i}$ where $v_{i}=\overline{\hat{\sigma}^{-1}\left(u_{i}\right)}$.

A nontrivial path $\gamma \subseteq \Gamma$ is a periodic Nielsen path for $\hat{\sigma}$ if for some $m>0$, we have $\left[\hat{\sigma}^{m}(\gamma)\right]=$ [ $\gamma]$. If $m=1$ we call $\gamma$ a Nielsen path. An exceptional path in $\Gamma$ is a path of the form $E_{i} \gamma^{m} \bar{E}_{j}$, where $\gamma$ is a primitive Nielsen path, and $\hat{\sigma}\left(E_{i}\right)=E_{i} \gamma^{p}$ and $\hat{\sigma}\left(E_{j}\right)=E_{j} \gamma^{q}$ for $p, q>0$ and any $m$. For a unipotent polynomially growing automorphism, every closed periodic Nielsen path is Nielsen [6, Proposition 3.16]. If $p \neq q$ we say the exceptional path is linearly growing, otherwise it is an exceptional Nielsen path.

Every path $\gamma \subseteq \Gamma$ has a canonical decomposition with respect to an upper triangular $\hat{\sigma}$ into single edges and maximal exceptional paths [6, Lemma 4.26].

For all of the terms in the previous two paragraphs, when we are dealing with more than one upper-triangular homotopy equivalence we will specify which homotopy equivalence is involved, e.g. "a path $\gamma$ is $\hat{\sigma}$-Nielsen" or "consider the $\hat{\tau}$-canonical decomposition of $\gamma=\gamma_{1} \gamma_{2} \cdots \gamma_{k}$ ".

The analogy between unipotent polynomially growing outer automorphisms and unipotent matrices stretches beyond having an upper-triangular basis. The classical Kolchin theorem for linear groups 23 states that if a subgroup $H \leq G L(n, \mathbb{C})$ consists of unipotent matrices then there is a basis so that with respect to this basis every element of $H$ is upper triangular with 1 's on the diagonal. There is an analogous theorem for unipotent polynomially growing outer automorphisms, due to Bestvina, Feighn, and Handel.

Theorem 3.2 [6, Main Theorem]. Suppose $H \leq \operatorname{Out}\left(F_{n}\right)$ is a finitely generated subgroup with every element unipotent polynomially growing. Then there is a filtered graph $\Gamma$ and a fixed preferred orientation such that every $\sigma \in H$ is upper triangular with respect to $\Gamma$. 
Remark 3.3. Bestvina, Feighn, and Handel use a different definition of upper-triangular, allowing that $\sigma\left(E_{i}\right)=v_{i} E_{i} u_{i}$, however our definition can be obtained by subdividing each edge and doubling the length of the filtration.

\subsection{Dehn twists and linear growth}

Let $\Sigma$ be a closed hyperbolic surface. Given $\gamma \subseteq \Sigma$ an essential simple closed curve, consider a homeomorphism $\tau_{\gamma}: \Sigma \rightarrow \Sigma$ that is the identity outside an annular neighborhood of $\gamma$ and performs a twist of $2 \pi$ on the annulus. Such a homeomorphism is known as a Dehn twist. The induced map $\tau_{\gamma *}: \pi_{1}(\Sigma) \rightarrow \pi_{1}(\Sigma)$ can be expressed in terms of the graph of groups decomposition of $\pi_{1}(\Sigma)$ induced by $\gamma$, and this expression motivates the following definition for general graphs of groups.

Definition 3.4. Suppose $\Gamma$ is a graph of groups. Given a fixed collection of edges $\left\{e_{i}\right\} \subseteq$ $E(\Gamma)$ closed under the edge involution and $z_{e_{i}} \in Z\left(G_{e_{i}}\right)$ satisfying $z_{\bar{e}_{i}}=z_{e_{i}}^{-1}$, the Dehn twist about $\left\{e_{i}\right\}$ by $\left\{z_{i}\right\}, D_{z} \in \operatorname{Out}\left(\pi_{1}(\Gamma, v)\right)$, is the outer automorphism induced by $\tilde{D}_{z}$ on the fundamental groupoid of $\Gamma$, given by

$$
\begin{aligned}
\tilde{D}_{z}\left(e_{i}\right) & =e_{i} z_{i}^{e_{i}} & \\
\tilde{D}_{z}(g) & =g, & g \in G_{v}, v \in V(\Gamma) \\
\tilde{D}_{z}(e) & =e, & e \notin\left\{e_{i}\right\}
\end{aligned}
$$

The induced outer automorphism does not depend on the choice of basepoint.

Note that $D_{z}^{n}=D_{z^{n}}$, defining $z^{n}=\left\{z_{e_{i}}^{n}\right\}$ for any $n$, and that any two twists on a fixed graph of groups $\Gamma$ commute. The requirement that each $z_{e_{i}} \in Z\left(G_{e_{i}}\right)$ is necessary to ensure that the defining relations of the fundamental groupoid are respected. In turn, when $\pi_{1}(\Gamma, v)$ is free a Dehn twist can only twist around edges with cyclic stabilizers.

ExAMPLE 3.5. Let $\Gamma$ be the graph of groups associated to the amalgamated product $A *_{C} B$ and $z \in Z(C)$. The twist of $\Gamma$ about its edge by $z$ can be represented by $D_{z}(a)=z^{-1} a z, a \in A$, $D_{z}(b)=b, b \in B$. Since $A \cup B$ generates $\pi_{1}(\Gamma, v)$ this fully specifies the automorphism.

Let $\mathcal{H}$ be the graph of groups associated to the HNN extension $A *_{C}$ and pick $z \in Z(C)$. The twist of $\mathcal{H}$ about its one edge by $z$ is represented by $D_{z}(a)=a$ and $D_{z}(t)=t z$ with $a \in A$ and $t$ the edge of the extension.

Specializing these examples to splittings of $\pi_{1}(\Sigma)$ given by an essential closed curve in a closed hyperbolic surface $\gamma \subseteq \Sigma$, this gives the previously mentioned algebraic representation of $\tau_{\gamma^{*}}$ as the Dehn twist about the edge of the splitting corresponding to $\gamma$ by $\gamma_{*} \in \pi_{1}(\Sigma)$.

EXAMPLE 3.6 Nielsen automorphisms of $F_{r}$. Consider the graph of groups $\Gamma$ in Figure 3. The edge morphisms for the single edge are given by $\iota_{t}(z)=a_{j}$ and $\iota_{\bar{t}}(z)=a_{k}$. The map $F:\left\langle x_{1}, \ldots, x_{n}\right\rangle \rightarrow \pi_{1}(\Gamma, v)$ given by $F\left(x_{i}\right)=a_{i}, i \neq j$, and $F\left(x_{j}\right)=t$ gives a realization of the Nielsen automorphism $\phi\left(x_{i}\right)=x_{i}, \phi\left(x_{j}\right)=x_{k} x_{j}$ as the Dehn twist about the single edge by $z$.

A Dehn twist outer automorphism has many graph of groups representatives, most of which are not well suited to analysis using the Guirardel core, due to lots of extra information. Certain ill-behaved stabilizers, non-minimal graphs, invisible vertices, and unused edges all cause trouble. Cohen and Lustig identified a particularly useful class of representatives, called efficient twists. 


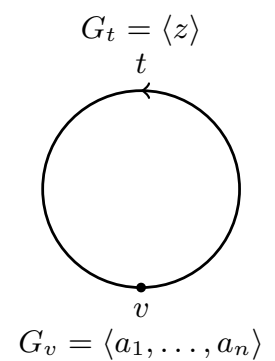

FiguRE 3. The graph of groups used to represent Nielsen automorphisms.

Definition 3.7. A Dehn twist $D$ on a graph of groups $\Gamma$ is efficient if

(i) $\Gamma$ is minimal, small, and visible,

(ii) $D$ twists about every edge (every $z_{e} \neq$ id),

(iii) (no positively bonded edges) there is no pair of edges $e_{1}, e_{2} \in E(\Gamma)$ such that $v=t\left(e_{1}\right)=$ $t\left(e_{2}\right)$, and integers $m, n \neq 0$ with $m n>0$, such that $z_{e_{1}}^{m}$ is conjugate in $G_{v}$ to $z_{e_{2}}^{n}$.

Cohen and Lustig remark that it is a consequence of these three properties that $\Gamma$ is necessarily very small. Returning our attention to $\operatorname{Out}\left(F_{r}\right)$ a Dehn twist outer automorphism $D \in \operatorname{Out}\left(F_{r}\right)$ is one that can be represented as a Dehn twist of some graph of groups decomposition of $F_{r}$ (such a decomposition necessarily only twists about those edges with cyclic edge groups). These outer automorphisms have linear growth (and all outer automorphisms with linear growth are roots of Dehn twists [25]).

By assigning each edge of a graph of groups $\Gamma$ a positive length, the Bass-Serre tree $T$ of $\Gamma$ becomes a metric $F_{r}$-tree. Given a very small graph of groups $\Gamma$ with fundamental group $F_{r}$, the collection of projective classes of all choices of metric on $T$ determines an open simplex $\Delta(\Gamma) \subseteq$ $\overline{C V}_{r}$ in projectivized outer space. If $\Gamma$ is visible and minimal, this simplex is of dimension $|E(\Gamma)|-1$. When $D$ is an efficient Dehn twist on $\Gamma$, the simplex $\Delta(\Gamma)$ is completely determined by the dynamics of the action of $D$ on $C V_{r}$, as shown by Cohen and Lustig [10].

Theorem 3.8 [10, Theorem 13.2]. Suppose $D$ is a Dehn twist in $\operatorname{Out}\left(F_{r}\right)$ with an efficient representative on a graph of groups $\Gamma$. Then for all $[T] \in C V_{r}$,

$$
\lim _{n \rightarrow \infty} D^{n}([T])=\lim _{n \rightarrow \infty} D^{-n}([T]) \in \Delta(\Gamma) .
$$

Corollary 3.9. If $D \in \operatorname{Out}\left(F_{r}\right)$ has an efficient Dehn twist representative, then the simplicial structure of the Bass-Serre tree of the representative is unique.

Proof. Suppose $D$ has efficient representatives $D_{1}$ on $\Gamma_{1}$ and $D_{2}$ on $\Gamma_{2}$. By the theorem, $\Delta\left(\Gamma_{1}\right)=\Delta\left(\Gamma_{2}\right)$ since two open simplices which share a point are equal. This completes the claim.

The efficient graph of groups representative of a Dehn twist can be constructed from an upper-triangular representation. Bestvina, Feighn, and Handel give this construction in the metric category, using a particular upper-triangular representation that permits them to compute metric information about the limit in $\overline{C V}_{r}$, but the uniqueness of the algebraic structure permits the calculation from any upper-triangular representation. First note that 
an upper-triangular homotopy equivalence grows linearly if and only if each suffix is Nielsen, and that each edge is either fixed or grows linearly.

To construct the efficient representative from an upper-triangular representative we need the notion of folding in a tree or graph, due to Stallings [30. In a simplicial $F_{r}$-tree $T$, a fold of two edges $u, v \in T$ with $o(u)=o(v)$ for a linear homeomorphism $\phi: u \rightarrow v$ is the quotient of $T$ by the smallest equivalence relation satisfying $x \sim \phi(x)$ for all points $x \in u$ and if $x \sim y$ and $g \in F_{r}$ then $x . g \sim y . g$. The quotient map of this equivalence $\tilde{f}: T \rightarrow T / \sim$ is called the folding map, and the resulting space $T / \sim$ is a $F_{r}$-tree (it may be necessary to subdivide to ensure that the action is without inversions). When the action on the folded tree $T / \sim$ is without inversions, we get a graph of groups morphism on the quotient $f: \bar{T} \rightarrow \overline{T / \sim}$.

Let $q: T \rightarrow \bar{T}$ be a graph of groups quotient map. There is a particular type of fold we treat in detail. Suppose there is an element $g \in G$ such that the folding homeomorphism $\phi: u \rightarrow v$ is induced by the $g$ action. In this case $g \in \bar{T}_{o(u)}$ and $g$ conjugates $\operatorname{Stab}(u)$ to $\operatorname{Stab}(v)$. The folded graph of groups $\overline{T / \sim}$ has the same combinatorial structure as $\bar{T}$, however $\operatorname{Stab}(u / \sim)=$ $\langle\operatorname{Stab}(u), g\rangle$, so that $u / \sim$ has a larger edge group. This is referred to as "pulling an element in a vertex group over an edge".

By subdividing an edge we may perform a partial fold of the first half of $u$ over $v$. (Partial folding can be discussed in much greater generality; we require only the midpoint version.) We will often specify a fold by a pair of edges $u$ and $v$ with $o(u)=o(v)$ in the quotient graph of groups, it is understood that we mean the equivariant fold of all pairs of lifts $\tilde{u}, \tilde{v}$ with $o(\tilde{u})=o(\tilde{v})$. The definition of folding generalizes to allow $v$ to be an edge path, and we use this more general definition.

Lemma 3.10. Suppose $\hat{\sigma}: \Gamma \rightarrow \Gamma$ is a linearly growing upper-triangular homotopy equivalence of a filtered graph $\Gamma$. Then there is an $F_{r}$-tree $T$ and a composition of folds and collapses $f: \tilde{\Gamma} \rightarrow T$ which realizes the outer automorphism represented by $\hat{\sigma}$ as an efficient Dehn twist on the graph of groups quotient $\bar{T}$.

Proof. The strategy of the proof is to collapse every fixed edge; in the resulting graph of groups, the suffix of the lowest linear edge is in a vertex group, and so the suffix can be folded over that edge. Working up the filtration in this fashion the result is a graph of groups with cyclic edge stabilizers, and by twisting on every edge by the twister specified by its suffix; the result is a Dehn twist on this graph which represents $\hat{\sigma}$.

The problem with this construction, as just described, is that the result may not be efficient: there may be obtrusive powers, and there may be positively bonded edges. The first problem is solved by using the primitive root of the suffix, but the second requires some work. One could use Cohen and Lustig's algorithm to remove positive bonding, however we give a different construction similar to that of Bestvina, Feighn, and Handel [6] useful when considering more than one Dehn twist. In this construction we first fold certain edges with related suffix so that when we carry out the sketch above no positive bonding results.

We assume without loss of generality $\Gamma$ is minimal (that is, the quotient of a minimal tree under the $F_{r}$ action).

Step 1: Fold Conjugates. We construct a series of folds by working up the filtration from lowest edge to highest. Start with $\Gamma^{0}=\Gamma$. Suppose the suffix $u_{i}$ of $E_{i}$ is of the form $\gamma_{i}\left[\eta_{j}^{k}\right] \bar{\gamma}_{i}$ with $k \neq 0$, where $u_{j}=\left[\eta_{j}^{k^{\prime}}\right]$ so that $\eta_{j}$ is the primitive Nielsen path associated to $u_{j}, j<i$ and $\gamma_{i}$ a closed path of height at most $i-1$. Since $u_{i}$ is Nielsen and tight we must have $\left[\hat{\sigma}_{i-1}\left(\gamma_{i}\right)\right]=\gamma_{i} \eta_{j}^{m}$ for some $m \in \mathbb{Z}$ (possibly zero). In this case fold the terminal half of $E_{i}$ over $\bar{\gamma}_{i}$. Let $f_{i}: \Gamma^{i-1} \rightarrow \Gamma^{i}$ be the folding map in this step. We claim the induced homotopy equivalence satisfying $\hat{\sigma}_{i} f_{i}=f_{i} \hat{\sigma}_{i-1}$ has an upper triangular tightening. Let $E_{i}^{\prime}$ denote the 
unfolded initial half of $E_{i}$, and filter $\Gamma^{i}$ by the filtration of $\Gamma^{i-1}$ where the $i$ th stratum is now $E_{i}^{\prime}$. It suffices to check that $\hat{\sigma}_{i}\left(E_{i}^{\prime}\right)=E_{i}^{\prime} u_{i}^{\prime}$. Indeed, using the equation

$$
f_{i} \hat{\sigma}_{i-1}\left(E_{i} \gamma_{i}\right)=\hat{\sigma}_{i}\left(E_{i}^{\prime}\right)
$$

we have for some $m \in \mathbb{Z}$

$$
f_{i} \hat{\sigma}_{i-1}\left(E_{i} \gamma_{i}\right)=f_{i}\left(E_{i} \gamma_{i} \eta_{j}^{k} \bar{\gamma}_{i} \gamma_{i} \eta_{j}^{m}\right)=E_{i}^{\prime} \bar{\gamma}_{i} \gamma_{i} \eta_{j}^{k} \bar{\gamma}_{i} \gamma_{i} \eta_{j}^{m}
$$

and so the tightening of $\hat{\sigma}_{i}$ gives $E_{i}^{\prime}$ suffix $\left[\eta_{j}^{k+m}\right]$ (for edges other than $E_{i}^{\prime}$ the suffix is the same as that of $\hat{\sigma}_{i-1}$, which already has upper triangular tightening). If the suffix $u_{i}$ of $E_{i}$ is not of the above form, take $\Gamma^{i}=\Gamma^{i-1}$ and $f_{i}=\mathrm{id}$.

Denote the total folding map $f_{k} \cdots f_{0}=f^{\prime}: \Gamma \rightarrow \Gamma^{\prime}$, and the tightening of the induced automorphism $\hat{\sigma}^{\prime}$. By construction $\hat{\sigma}^{\prime}$ is upper triangular and has the property that for every two edges $E_{i}$ and $E_{j}$ with common terminal vertex, if their suffixes have conjugate roots then they are of the form $u_{i}=\left[\eta^{k_{i}}\right], u_{j}=\left[\eta^{k_{j}}\right]$ for positive powers of a primitive Nielsen path $\eta$.

Step 2: Fold Linear Families. Starting now with $\hat{\sigma}^{\prime}$, we perform another sequence of folds to ensure that twisters will not be positively bonded. For a primitive Nielsen path $\eta$, the linear family associated to $\eta$ is all edges of $\Gamma^{\prime}$ with suffix $\left[\eta^{k}\right]$ for some $k \neq 0$. We now work down the filtration of $\Gamma^{\prime}$. Set $\Gamma_{k}^{\prime}=\Gamma^{\prime}$. If $E_{i}^{\prime}$ is in the linear family associated to some primitive Nielsen path $\eta$, let $E_{j}$ be the next edge lower than $E_{i}^{\prime}$ in the linear family, and fold half of $E_{i}^{\prime}$ over all of $E_{j}^{\prime}$. Denote the fold $f_{i}^{\prime}: \Gamma_{i}^{\prime} \rightarrow \Gamma_{i-1}^{\prime}$ in this case; otherwise set $\Gamma_{i-1}^{\prime}=\Gamma_{i}$ and $f_{i}^{\prime}=\mathrm{id}$. Let $\Gamma^{\prime \prime}=\Gamma_{0}^{\prime}$ be the total result of this folding, with total folding map $f_{0}^{\prime} \cdots f_{k}^{\prime}=f^{\prime \prime}: \Gamma^{\prime} \rightarrow \Gamma^{\prime \prime}$, and denote the unfolded halves of edges by $E_{i}^{\prime \prime}$. (If an edge is not folded we will also use $E_{i}^{\prime \prime}$ for the edge as an edge of $\left.\Gamma^{\prime \prime}\right)$. The graph $\Gamma^{\prime \prime}$ is naturally filtered, with the filtration induced by $f^{\prime \prime}$. We claim that the induced homotopy equivalence $\hat{\sigma}^{\prime \prime}=f^{\prime \prime} \hat{\sigma}^{\prime} f^{\prime \prime-1}$ is again upper triangular. Indeed, as in the previous case we can calculate the suffixes. For $E_{i}^{\prime}$ denote by $E_{i_{1}}^{\prime}, \ldots E_{i_{l}}^{\prime}$ the edges in the linear family of $E_{i}^{\prime}$ below $E_{i}^{\prime}$ in descending order, so that $f^{\prime \prime}\left(E_{i}^{\prime}\right)=E_{i}^{\prime \prime} E_{i_{1}}^{\prime \prime} \cdots E_{i_{l}}^{\prime \prime}$. Working inductively up the linear family, a calculation similar to the previous step finds $\hat{\sigma}^{\prime \prime}\left(E_{i}^{\prime \prime}\right)=E_{i}^{\prime \prime} E_{i_{1}}^{\prime \prime} \cdots E_{i_{l}}^{\prime \prime}\left[f^{\prime \prime}(\eta)^{k_{i}-k_{i_{1}}}\right] \bar{E}_{i_{l}}^{\prime \prime} \cdots \bar{E}_{i_{1}}^{\prime}$, and the associated primitive Nielsen path to $E_{i}^{\prime \prime}$ is $\eta_{i}^{\prime \prime}=E_{i_{1}}^{\prime \prime} \cdots E_{i_{l}}^{\prime \prime}\left[f^{\prime \prime}(\eta)\right] \bar{E}_{i_{l}}^{\prime \prime} \cdots \bar{E}_{i_{1}}^{\prime \prime}$.

Step 3: Collapse and Fold Edge Stabilizers. From $\hat{\sigma}^{\prime \prime}$ and $\Gamma^{\prime \prime}$ we can now construct a graph of groups; the previous two steps will ensure that no twisters in the result are positively bonded. We work up the filtration once more. Let $\bar{T}^{0}$ be the graph of groups constructed from $\Gamma^{\prime \prime}$ by collapsing all edges with trivial suffix. Obtain $\bar{T}^{i}$ from $\bar{T}^{i-1}$ as follows. If $\hat{\sigma}^{\prime \prime}\left(E_{i}^{\prime \prime}\right)=E_{i}^{\prime \prime}$, set $\bar{T}^{i}=\bar{T}^{i-1}$. If $\hat{\sigma}^{\prime \prime}\left(E_{i}^{\prime \prime}\right)=E_{i}^{\prime \prime}\left[\eta_{i}^{\prime \prime} k_{i}^{\prime \prime}\right]$ then obtain $\bar{T}^{i}$ from $\bar{T}^{i}$ by pulling $\eta_{i}^{\prime \prime}$ over $E_{i}^{\prime \prime}$. By construction $\eta_{i}^{\prime \prime}$ represents an element in a vertex group at some lift $t\left(E_{i}^{\prime \prime}\right)$. The result is $\bar{T}$. The composition of folding maps $f^{\prime \prime}: \Gamma^{\prime \prime} \rightarrow \bar{T}$ induces a Dehn twist $\tilde{\sigma}$ on $\bar{T}$ where the system of twisters is given by $z_{E_{i}^{\prime \prime}}=\eta_{i}^{\prime \prime} k_{i}^{\prime \prime}$. By construction, this twist represents $\hat{\sigma}^{\prime \prime}$ and so $\hat{\sigma}$; moreover the edge stabilizers are not conjugate in the vertex groups, as a result of the first two steps; therefore the resulting twist is efficient except for the possibility of invisible vertices. Invisible vertices are an artifact of the graph of groups; removing them gives the desired efficient twist.

REMARK 3.11. It is possible that $\hat{\sigma}$ is upper triangular with respect to several different filtrations of $\Gamma$. By fixing a filtration a choice is being made, but the choices made do not matter because of Corollary 3.9 . 
EXAmple 3.12. To illustrate the procedure in Lemma 3.10 we calculate the efficient representative of $\sigma \in \operatorname{Out}\left(F_{4}\right)$ given by

$$
\begin{aligned}
a & \mapsto a d b c b^{-1} d^{-1} \\
b & \mapsto b c \\
c & \mapsto c \\
d & \mapsto d .
\end{aligned}
$$

We will start with the upper triangular representative $\hat{\sigma}: \Gamma \rightarrow \Gamma$ on the rose on 4 petals with topological edges named $a, b, c, d$ filtered by reverse alphabetical order and the images of edges under $\hat{\sigma}$ given as above. This representative has a single linear family $\{a, b\}$ with associated primitive Nielsen path $c$.

Step 1: Fold Conjugates. Working up the filtration we find that the only edge that needs folding is $a$, we fold half of $a$ over $\bar{b} \bar{d}$. This gives the folding map $f^{\prime}: \Gamma \rightarrow \Gamma^{\prime}$ where $\Gamma^{\prime}$ is a rose on four petals with edges $\left(a^{\prime}, b, c, d\right), f^{\prime}(a)=a^{\prime} \bar{b} \bar{d}$, and $f^{\prime}(e)=e$ for $e \neq a$. The induced upper triangular representative $\hat{\sigma}^{\prime}: \Gamma^{\prime} \rightarrow \Gamma^{\prime}$ is given by

$$
\begin{aligned}
a^{\prime} & \mapsto a^{\prime} c^{2} \\
b & \mapsto b c \\
c & \mapsto c \\
d & \mapsto d .
\end{aligned}
$$

Indeed, we can verify that $\hat{\sigma}^{\prime}\left(a^{\prime}\right)=a^{\prime} c^{2}$ by calculating:

$$
\hat{\sigma}^{\prime}\left(a^{\prime}\right)=\hat{\sigma}^{\prime}\left(a^{\prime} \bar{b} \bar{d} d b\right)=\hat{\sigma}^{\prime}(f(a d b))=[f(\hat{\sigma}(a d b))]=\left[a^{\prime} \bar{b} \bar{d} d b c^{2}\right]=a^{\prime} c^{2} .
$$

Step 2: Fold Linear Families. Working down the filtration, the only edge that requires folding is $a^{\prime}$ : we fold the terminal half over $b$. This defines $f^{\prime \prime}: \Gamma^{\prime} \rightarrow \Gamma^{\prime \prime}$ where $\Gamma^{\prime \prime}$ is the four petals with edges $\left(a^{\prime \prime}, b, c, d\right), f^{\prime \prime}\left(a^{\prime}\right)=a^{\prime \prime} b$, and $f^{\prime \prime}(e)=e$ for $e \neq a^{\prime}$. Calculating $\hat{\sigma}^{\prime \prime}\left(a^{\prime \prime}\right)$ in a similar fashion:

$$
\hat{\sigma}^{\prime \prime}\left(a^{\prime \prime}\right)=\left[f^{\prime \prime}\left(\hat{\sigma}^{\prime}\left(a^{\prime} b\right)\right)\right]=\left[a^{\prime \prime} b c^{2} \bar{c} \bar{b}\right]=a^{\prime \prime} b c \bar{b} .
$$

The action of $\hat{\sigma}^{\prime \prime}$ on the remaining edges is the same as that of $\hat{\sigma}^{\prime}$.

Step 3: Collapse and Fold Edge Stabilizers. Once more working up the filtration we first collapse the edges $c$ and $d$ with trivial suffix, which gives the graph of groups $\bar{T}^{0}$ which has two free edges $a^{\prime \prime}$ and $b$, and a vertex with stabilizer $\langle c, d\rangle$.

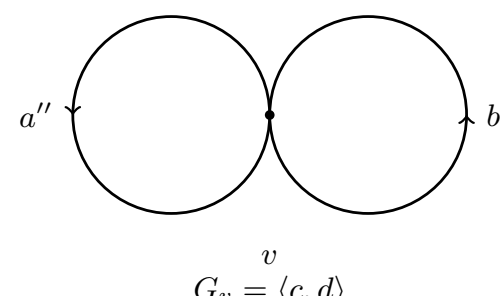

Next, we pull $c$ over $b$, and (using the orientation $\{\bar{a}, \bar{b}\}$ and keeping in mind that in this article we are using right actions) obtain $\bar{T}^{1}$. 


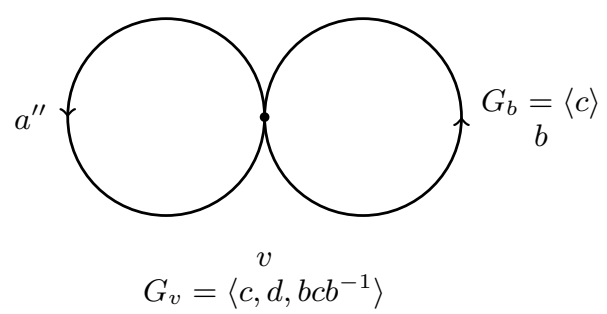

Finally, we pull $b c \bar{b}$ over $a^{\prime \prime}$ and using the same orientation for labels arrive at $\bar{T}$.

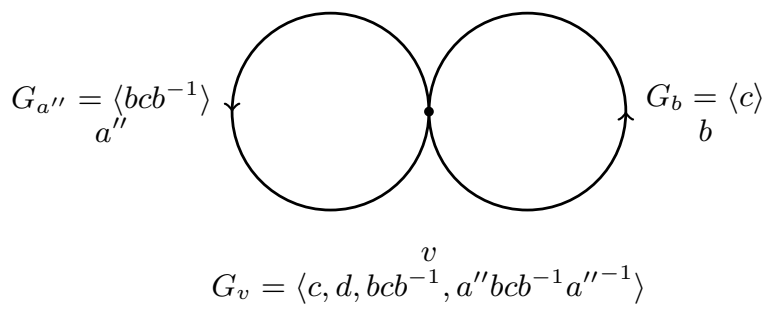

The Dehn twist representative $\tilde{\sigma}$ is given by the system of twisters $z_{a^{\prime \prime}}=b c b^{-1} z_{b}=c$. Observe $a^{\prime \prime}=a d$.

The upper triangular representative constructed in the previous lemma provides us with a basis of $F_{r}$ with small bounded cancellation constant for the length function on the Bass-Serre tree $T$.

Lemma 3.13. Suppose $\sigma$ is an efficient Dehn twist on the very small graph of groups $\bar{T}$, and let $T$ be the Bass-Serre tree. Then there is a basis $\Lambda$ for $F_{r}$ such that the bounded cancellation constant constant $C(\Lambda, T)$ from Lemma 2.7 satisfies

$$
C(\Lambda, T) \leq 6 r(2 r-2)
$$

Proof. From Lemma 2.7, we know that $C(\Lambda, T) \leq 6 r \operatorname{Lip}(f)$ for any Lipshitz surjection $f: S_{\Lambda} \rightarrow T$, where $S_{\Lambda}$ is the universal cover of a wedge of circles marked by $\Lambda$. Therefore it suffices to produce an $F_{r}$-tree $S$ with quotient a wedge of $r$ circles and a map $f: S \rightarrow T$ so that $\operatorname{Lip}(f) \leq 2 r-2$. The basis corresponding to the circles in the quotient of $S$ is then the desired basis.

By Lemma 3.10 there is a simplicial tree $\Gamma^{\prime \prime}$ and a map $f: \Gamma^{\prime \prime} \rightarrow T$ that is a composition of folds and collapses. Thus the map $f: \Gamma^{\prime \prime} \rightarrow T$ has Lipshitz constant 1 . The tree $\Gamma^{\prime \prime}$ is equivalent to one with no valence one or two vertices so $\Gamma^{\prime \prime} / F_{r}$ has at most $3 r-3$ edges. By fixing a maximal tree $K \subseteq \Gamma^{\prime \prime} / F_{r}$, the collapse of this maximal tree gives a wedge of circles $R$ with $r$ edges, and a homotopy equivalence $g: R \rightarrow \Gamma^{\prime \prime} / F_{r}$ with Lipshitz constant at most $\operatorname{diam}(K) \leq 2 r-2$. The composition of the lift $\tilde{g}$ with $f$ gives $f \circ \tilde{g}: \tilde{R} \rightarrow T$, which is the desired map. 


\section{Guiding examples}

When the Guirardel core of two Bass-Serre trees has no rectangles, its quotient provides a simultaneous resolution of the two graphs of groups. This construction immediately gives us a sufficient condition for two Dehn twists to commute.

Lemma 4.1. Suppose $\tilde{\sigma}$ and $\tilde{\tau}$ are efficient Dehn twists based on graphs of groups $\bar{A}$ and $\bar{B}$ covered by $F_{r}$-trees $A$ and $B$ respectively, representing $\sigma, \tau \in \operatorname{Out}\left(F_{r}\right)$. If $i(A, B)=0$ then $[\sigma, \tau]=1$ in $\operatorname{Out}\left(F_{r}\right)$.

Proof. Since $A$ and $B$ are simplicial, $i(A, B)=0$ implies that $\widehat{\mathcal{C}}(A, B)$ is a tree. Therefore, by Lemma 2.21, $\widehat{\mathcal{C}}(A, B)$ is the Bass-Serre tree of a graph of groups $\Gamma$, and we may without loss of generality assume $\bar{A}$ and $\bar{B}$ fit into the following diagram, where $\pi_{\bar{A}}$ and $\pi_{\bar{B}}$ are quotient graph of groups morphisms that collapse edges.

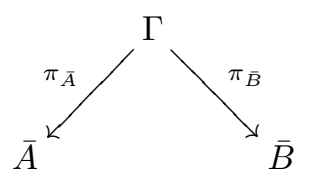

Moreover (and this is still the content of Lemma 2.21), the edge groups of $\Gamma$ are edge groups of either $\bar{A}$ or $\bar{B}$.

Define $\hat{\sigma}$ on $\Gamma$ by the system of twisters

$$
z_{e}=\left\{\begin{array}{cc}
z_{\pi_{\bar{A}}(e)} & \pi_{\bar{A}}(e) \in E(\bar{A}) \\
1 & \text { otherwise }
\end{array}\right.
$$

By construction, $\pi_{\bar{A}} \hat{\sigma}=\tilde{\sigma} \pi_{\bar{A}}$ at the level of the fundamental groupoid, so that $\hat{\sigma}$ is also a representative of $\sigma$. (The induced automorphism on the fundamental group coming from a graph of groups collapse is the identity [10.) Similarly define $\hat{\tau}$, thus simultaneously realizing $\sigma$ and $\tau$ as Dehn twists on $\Gamma$, whence $[\sigma, \tau]=1$.

Towards a converse, Clay and Pettet give a partial result, using the notion of a filling pair of Dehn twists 9 . The other key tool is the ping-pong lemma, which we use in the following formulation similar to the form used by Clay and Pettet and Hamidi-Tehrani $[9,18$.

Lemma 4.2 Ping-Pong. Suppose $G=\langle a, b\rangle$ acts on a set $P$, and there is a partition $P=$ $P_{a} \sqcup P_{b}$ into disjoint subsets such that $a^{ \pm n}\left(P_{b}\right) \subseteq P_{a}$ and $b^{ \pm n}\left(P_{a}\right) \subseteq P_{b}$ for all $n>0$. Then $G \cong F_{2}$.

Proof. Any non-trivial reduced word is either a power of $a$ or conjugate to one of the form $w=a^{n_{1}} w^{\prime} a^{n_{2}}$ for non-zero integers $n_{1}, n_{2}$ and $w^{\prime}$ reduced starting and ending with a power of $b$. For $w$ in this form, $w\left(P_{b}\right) \cap P_{b} \subseteq P_{a} \cap P_{b}=\emptyset$, so $w \neq$ id.

Definition 4.3. Let $X$ be a finitely generated group acting on $T$ a simplicial tree. The free $T$ volume of $X, \operatorname{covol}_{T}(X)$ is the number of edges with trivial stabilizer in the graph of groups quotient of the minimal subtree $T^{X} \subset T$.

Note that $\operatorname{covol}_{T}(\langle g\rangle)=\ell_{T}(g)$ for $g \in X$. 
Definition 4.4. Two graphs of groups $\bar{A}$ and $\bar{B}$ associated to $F_{r}$-trees $A$ and $B$ fill if for every proper free factor or infinite cyclic subgroup $X \leq F_{r}$,

$$
\operatorname{covol}_{A}(X)+\operatorname{covol}_{B}(X)>0 .
$$

Definition 4.5. Suppose $\tilde{\sigma}, \tilde{\tau}$ are representatives of Dehn twists based on $\bar{A}$ and $\bar{B}$, where both graphs of groups have one edge and fundamental group $F_{r}$. If $\bar{A}$ and $\bar{B}$ fill then we call the induced outer automorphisms $\sigma$ and $\tau$ a filling pair.

This definition is a close parallel to the notion of a pair of filling simple closed curves, and Clay and Pettet strengthen this parallel to a theorem.

Theorem 4.6 [9, Theorem 5.3]. Suppose $\sigma, \tau \in \operatorname{Out}\left(F_{r}\right)$ are a filling pair of Dehn twists. Then there is an $N$ such that

(i) $\left\langle\sigma^{N}, \tau^{N}\right\rangle \cong F_{2}$ 母

(ii) If $\phi \in\left\langle\sigma^{N}, \tau^{N}\right\rangle$ is not conjugate to a generator then $\phi$ is an atoroidal fully irreducible outer automorphism.

In developing their definition of free volume, Clay and Pettet use the Guirardel core as motivation, but give a form suited explicitly to the proof of their theorem. The definition of filling is indeed noticed by the core.

Proposition 4.7. Suppose $\bar{A}$ and $\bar{B}$ are small graphs of groups with one edge and fundamental group $F_{r}$ that fill. Then the Bass-Serre trees $A$ and $B$ have $i(A, B)>0$ and the action of $F_{r}$ on $\mathcal{C}(A, B)$ is free.

Proof. First, for any $(p, q) \in \mathcal{C}$, and $x \neq \mathrm{id} \in F_{r}$, we have $\ell_{A}(x)=\operatorname{covol}_{A}(<x>)$ and $\ell_{B}(x)=\operatorname{covol}_{B}(<x>)$. Since $\bar{A}$ and $\bar{B}$ fill,

$$
\ell_{A}(x)+\ell_{B}(x)=\operatorname{covol}_{A}(<x>)+\operatorname{covol}_{B}(<x>)>0
$$

and therefore $(p, q) \cdot x \neq(p, q)$.

To see that the core contains a rectangle we will show that the two trees have incompatible combinatorics (Lemma 2.20). To fix notation let $e$ be the edge of $\bar{A}$ and $f$ be the edge of $\bar{B}$. Let $\bar{A}_{e}=\langle c\rangle$. If $o(e) \neq t(e)$, let $a \in \bar{A}_{o(e)}$ be an element with no power conjugate into $\iota_{\bar{e}}\left(\bar{A}_{e}\right)$, and $\beta \in \bar{A}_{t(e)}$ be an element not conjugate into $\iota_{e}\left(\bar{A}_{e}\right)$. Set $b=e \beta e^{-1}$ in $\pi_{1}(\bar{A}, o(e))$. If $o(e)=t(e)$ take $a$ as before and $b=e$ in $\pi_{1}(\bar{A}, o(e))$.

By construction, $\ell_{A}(a b)>0$, and so $a b$ is not conjugate to $\iota_{\bar{e}}(c)$. Again, by the filling property, since $\ell_{A}(c)=0, \ell_{B}(c)>0$. Since $a b$ and $c$ are not conjugate, the characteristic sets of $a b$ and $c$ in $B$ meet in at most a finite number of edges of $C_{c}^{B}$, since $B$ is small. Thus there is some $n>0$ such that $C_{a b}^{B} \cap C_{c^{-n} a b c^{n}}^{B}=\emptyset$. However, by construction $C_{a b}^{A}$ contains the arc in $A$ stabilized by $c$, so $C_{a b}^{A} \cap C_{c^{-n} a b c^{n}}^{A^{n}}$ contains this arc for all $n$. Therefore the two Bass-Serre trees are incompatible, the core contains a rectangle, and since both trees are simplicial this implies that the intersection number is positive, as required.

\footnotetext{
$\dagger$ This conclusion holds under the weaker assumption that the two twists are hyperbolic-hyperbolic (Definition 4.8).
} 
This proposition motivates a variation of Clay and Pettet's result, in pursuit of a converse to Lemma 4.1. This variation cannot make the stronger assertion that the generated group contains an atoroidal fully irreducible element. Indeed, take $\sigma$ and $\tau$ to be a filling pair of Dehn twists for $F_{k}$ and consider the automorphism $\sigma * \mathrm{id}_{m}$ and $\tau * \mathrm{id}_{m}$ acting on $F_{k} * F_{m}$. This is a pair of Dehn twists of $F_{k+m}$ that has powers generating a free group, but does not fill, and every automorphism in $\left\langle\sigma * \mathrm{id}_{m}, \tau * \mathrm{id}_{m}\right\rangle$ fixes the conjugacy class of the complementary $F_{m}$ free factor, so all elements of the generated group represent reducible outer automorphisms. Nevertheless, there is a partial converse to Lemma 4.1 finding free groups generated by pairs of Dehn twists based on one-edge graphs of groups using a variation on their argument.

Definition 4.8. Suppose $\bar{A}$ and $\bar{B}$ are minimal visible small graphs of groups with one edge and associated $F_{r}$-trees $A$ and $B$. The pair is hyperbolic-hyperbolic if both for the edge $e \in E(\bar{A})$, a generator $z_{e}$ of $\bar{A}_{e}$ acts hyperbolically on $B$; and for the edge $f \in E(\bar{B})$, a generator $z_{f}$ of $\bar{B}_{f}$ acts hyperbolically on $A$.

Proposition 4.9. Suppose $\bar{A}$ and $\bar{B}$ are minimal visible small graphs of groups with one edge. If $\bar{A}$ and $\bar{B}$ are hyperbolic-hyperbolic, then $i(A, B)>0$.

Proof. The proof of Proposition 4.7 applies immediately to show that the two Bass-Serre trees are not compatible. The construction used only the positive translation length of $\ell_{B}(c)$ for a generator $c$ of an edge group of $\bar{A}_{e}$ and that $\bar{B}$ is small.

REMARK 4.10. As noted in the proof, the above proposition is much more general, giving a sufficient condition for incompatibility: for any two minimal, visible, small graphs of groups, if there is an edge of one with a generator hyperbolic in the other then the core of the Bass-Serre trees has a rectangle.

The hyperbolic-hyperbolic condition is sufficient to give a length function ping-pong argument similar to Clay and Pettet's.

Lemma 4.11. Suppose $\tilde{\sigma}$ and $\tilde{\tau}$ are efficient Dehn twist representatives of $\sigma, \tau \in \operatorname{Out}\left(F_{r}\right)$, on one-edge graphs of groups $\bar{A}$ and $\bar{B}$ respectively. If $\bar{A}$ and $\bar{B}$ are hyperbolic-hyperbolic, then for any $n \geq N=48 r^{2}-48 r+3$ the group $\left\langle\sigma^{n}, \tau^{n}\right\rangle \cong F_{2}$.

Proof. Let $e$ denote the edge of $\bar{A}, \bar{A}_{e}=\langle a\rangle, f$ the edge of $\bar{B}$ and $\bar{B}_{f}=\langle b\rangle$. Let $s, t$ be nonzero integers so that the twisters of $\tilde{\sigma}$ and $\tilde{\tau}$ are $z_{e}=a^{s}$ and $z_{f}=b^{t}$ respectively. We will conduct a ping-pong argument similar to Clay and Pettet's free factor ping pong technique. Consider the partitioned subset of conjugacy classes $P=P_{\sigma} \sqcup P_{\tau}$ defined by,

$$
\begin{aligned}
& P_{\sigma}=\left\{[w] \in P \mid \ell_{A}(w)<\ell_{B}(w)\right\} \\
& P_{\tau}=\left\{[w] \in P \mid \ell_{B}(w)<\ell_{A}(w)\right\} .
\end{aligned}
$$

This is a non-trivial partition, $a \in P_{\tau}$ and $b \in P_{\sigma}$ by hypothesis.

Our goal then is to find a power $N$ depending only on the rank such that for all $n \geq N$, $\sigma^{ \pm n}\left(P_{\tau}\right) \subseteq P_{\sigma}$ and $\tau^{ \pm n}\left(P_{\sigma}\right) \subseteq P_{\tau}$. By the ping-pong lemma, this implies $\left\langle\sigma^{n}, \tau^{n}\right\rangle \cong F_{2}$, as required. The argument will be symmetric. 
Suppose $[w] \in P_{\tau}$, so that $\ell_{A}(w)>0$. Fix a cyclically reduced representative in transverse Bass-Serre normal form with respect to an ordered basis $\Lambda$ of $F_{r}$ based at a vertex of $\bar{A}$ :

$$
w=e_{1} a^{k_{1}} w_{1} e_{2} a^{k_{2}} w_{2} \cdots e_{\ell} a^{k_{\ell}} w_{\ell}
$$

where $\ell=\ell_{A}(w), e_{i} \in\{e, \bar{e}\}$, we are suppressing the different edge morphisms sending $a$ into relevant vertex groups, and each $w_{i}$ is in the right transversal of the image of $a$ in the vertex group involved. Let $C$ be the bounded cancellation constant for the fixed basis of $F_{r}$ basis into $B$. With respect to this basis, after an appropriate conjugation we have the cyclically reduced conjugacy class representative $w^{\prime}$ satisfying

$$
\left|w^{\prime}\right|=\left|a^{k_{1}^{\prime}}\right|+\cdots+\left|w_{\ell-1}^{\prime}\right|+\left|a^{k_{\ell}^{\prime}}\right|+\left|w_{\ell}^{\prime}\right|
$$

where $w_{i}^{\prime}$ is the reduced word in this basis for the group element represented by the arrow $a^{ \pm 1} w_{i} e_{i+1} a^{ \pm 1}$ after collapsing a maximal tree, and $k_{i}^{\prime}$ differs from $k_{i}$ by a fixed amount depending only on the edges $e_{i}$ and $e_{i+1}$, as each where each $w_{i}^{\prime}$ might might disturb a fixed number of adjacent copies of conjugates of $a$ depending on the particular spelling (this follows from the minimality of the Schreier transversals used in transverse normal form). We have

$$
\ell_{A}(w)>\ell_{B}(w) \geq\left(\sum\left|k_{i}^{\prime}\right|\right) \ell_{B}(a)-2 C(\Lambda, B) \ell_{A}(w) .
$$

Re-writing, we conclude

$$
\sum\left|k_{i}^{\prime}\right|<\left(\frac{1+2 C(\Lambda, B)}{\ell_{B}(a)}\right) \ell_{A}(w) .
$$

Using the Dehn twist representative of $\sigma$, we calculate

$$
\tilde{\sigma}^{n}(w)=e_{1} a^{\epsilon_{1} s n} a^{k_{1}} w_{1} e_{2} a^{\epsilon_{2} s n} a^{k_{2}} w_{2} \cdots e_{\ell} a^{\epsilon_{\ell} s n} a^{k_{\ell}} w_{\ell}
$$

where $\epsilon_{i} \in\{ \pm 1\}$ according to the orientation of $e$ represented by $e_{i}$. Reducing these words, and applying bounded cancellation in the same fashion we have

$$
\begin{aligned}
\ell_{B}\left(\tilde{\sigma}^{n}(w)\right) & \geq \sum_{i=1}^{\ell}\left(\left|\epsilon_{i} s n+k_{i}^{\prime}\right|\right) \ell_{B}(a)-2 C(\Lambda, B) \ell_{A}(w) \\
& \geq\left(|s n| \ell_{A}(w)-\sum\left|k_{i}^{\prime}\right|\right) \ell_{B}(a)-2 C(\Lambda, B) \ell_{A}(w) \\
& \geq\left(|s n|-\frac{1+2 C(\Lambda, B)}{\ell_{B}(a)}\right) \ell_{A}(w) \ell_{B}(a)-2 C(\Lambda, B) \ell_{A}(w)
\end{aligned}
$$

with the last step following from Equation $\dagger$. Thus we have

$$
\frac{\ell_{B}\left(\tilde{\sigma}^{n}(w)\right)}{\ell_{A}\left(\tilde{\sigma}^{n}(w)\right)}=\frac{\ell_{B}\left(\tilde{\sigma}^{n}(w)\right)}{\ell_{A}(w)} \geq\left(|s n|-\frac{1+2 C(\Lambda, B)}{\ell_{B}(a)}\right) \ell_{B}(a)-2 C(\Lambda, B) .
$$

Therefore, to ensure $\sigma^{n}(w) \in P_{\sigma}$ we require

$$
\left(|s n|-\frac{1+2 C(\Lambda, B)}{\ell_{B}(a)}\right) \ell_{B}(a)-2 C(\Lambda, B)>1
$$

that is,

$$
|n|>\frac{2+4 C(\Lambda, B)}{|s| \ell_{B}(a)} .
$$

Since $|s| \ell_{B}(a) \geq 1$, having $|n|>2+4 C(\Lambda, B)$ suffices. By the Lemma 3.13 there is some basis $\Lambda$ such that $C(\Lambda, B) \leq 6 r(2 r-2)$, any choice of order on this basis will do. Let $N=48 r^{2}-$ $48 r+3$. The preceding calculation implies that for all $|n| \geq N, \sigma^{ \pm n}\left(P_{\tau}\right) \subseteq P_{\sigma}$. By a similar calculation, (using a good basis $\Lambda^{\prime}$ so that $C\left(\Lambda^{\prime}, A\right) \leq 6 r(2 r-2)$ ), we find that for any $|n| \geq N$, $\tau^{ \pm n}\left(P_{\sigma}\right) \subseteq P_{\tau}$. Therefore the group $\left\langle\sigma^{N}, \tau^{N}\right\rangle$ acting on $P=P_{\sigma} \sqcup P_{\tau}$ satisfies the hypotheses of the ping-pong lemma, and we conclude $\left\langle\sigma^{N}, \tau^{N}\right\rangle \cong F_{2}$ as required. 
REMARK 4.12. The reader familiar with Cohen and Lustig's skyscraper lemma and parabolic orbits theorem may wonder why these facts did not feature in the above proof. Both of these tools are not strong enough to give the uniform convergence necessary to carry out a pingpong type argument on $\overline{C V}_{r}$; the skyscraper lemma has constants that depend on the particular skyscraper involved, and the parabolic orbits theorem gives pointwise convergence of length functions on conjugacy classes but does not control the rate of convergence. A priori, this rate could be very bad, as demonstrated by the examples of Bestvina, Feighn, and Handel 6 , Remark 4.24].

Together Lemmas 4.1 and 4.11 come very close to a proof of Theorem 1.2 Nature is not so kind, and there are incompatible graphs of groups that are not hyperbolic-hyperbolic.

Example 4.13. Let $A$ and $C$ be the Bass-Serre trees of the following graphs of groups decompositions of $F_{3}$.
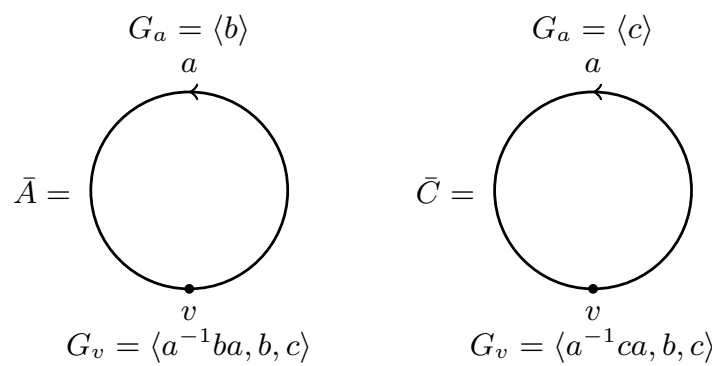

Let $\sigma$ and $\rho$ be the Nielsen transformations represented by Dehn twists about $\bar{A}$ and $\bar{C}$ by $b$ and $c$ respectively, so that

$$
\begin{aligned}
\sigma(a) & =b a & \rho(a) & =c a \\
\sigma(b) & =b & & \rho(b)=b \\
\sigma(c) & =c & \rho(c) & =c .
\end{aligned}
$$

We claim that $\mathcal{C}(A, C)$ has a rectangle, so that $i(A, C)>0$. Indeed, focus on the edges $e \subseteq A$ and $f \subseteq C$, each on the axis of $a$ with the induced orientation and the given edge stabilizers, illustrated below.
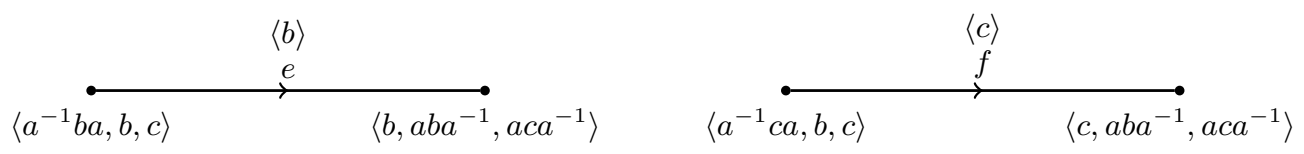

Note that $a \in \llbracket e \rrbracket \cap \llbracket f \rrbracket$ and $a^{-1} \in \llbracket \bar{e} \rrbracket \cap \llbracket \bar{f} \rrbracket$. Further, investigation of the diagrams shows that $b^{-1} a b \in \llbracket e \rrbracket \cap \llbracket \bar{f} \rrbracket$ and $c^{-1} a c \in \llbracket \bar{e} \rrbracket \cap \llbracket f \rrbracket$, so by Lemma 2.20 $e \times f \subseteq \mathcal{C}(A, B)$.

This example is not hyperbolic-hyperbolic; $\ell_{A}(c)=\ell_{C}(b)=0$. Nevertheless $\langle\sigma, \rho\rangle \cong F_{2}$. Indeed, $\omega \mapsto \omega(a) a^{-1}$ describes an isomorphism $\langle\sigma, \rho\rangle \cong\langle b, c\rangle$.

Example 4.14. Let $A$ and $B$ be the Bass-Serre trees of the following graphs of group decompositions of $F_{3}$. 

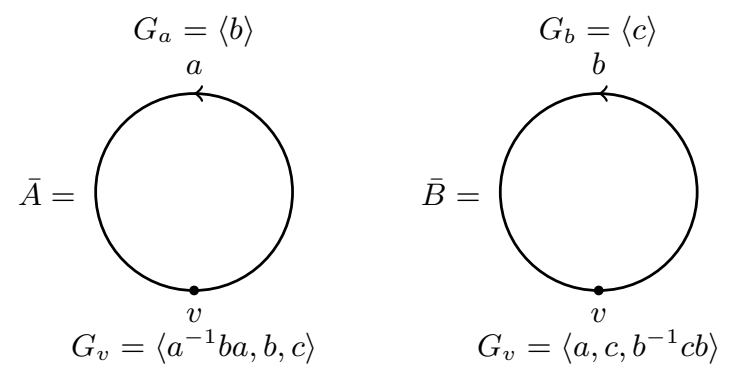

Let $\sigma$ and $\tau$ be the Nielsen transformations represented by Dehn twists about $\bar{A}$ and $\bar{B}$ by $b$ and $c$ respectively, so that

$$
\begin{aligned}
\sigma(a) & =b a & \tau(a) & =a \\
\sigma(b) & =b & & \tau(b)=c b \\
\sigma(c) & =c & \tau(c) & =c .
\end{aligned}
$$

Again we have a rectangle in $\mathcal{C}(A, B)$. Consider $g=a, h=b a b^{-1}$. Calculating with length functions we have

$$
\ell_{A}(g)=\ell_{A}(h)=1 \quad \ell_{B}(g)=\ell_{B}(h)=0
$$

and also

$$
\begin{gathered}
\ell_{A}(g h)=2 \neq 0=\ell_{A}\left(g h^{-1}\right) \\
\ell_{B}(g h)=\ell_{B}\left(g h^{-1}\right)=2>0=\ell_{B}(g)+\ell_{B}(h) .
\end{gathered}
$$

Therefore $A$ and $B$ do not have compatible combinatorics, so by Lemma $2.20 \mathcal{C}(A, B)$ has a rectangle and $i(A, B)>0$.

This example is also not hyperbolic-hyperbolic, $\ell_{B}(b)=1$ but $\ell_{A}(c)=0$. Again, however, $\left\langle\sigma^{3}, \tau^{3}\right\rangle \cong F_{2}$. For a ping-pong set we use $P=\left\{w a \in F_{3} \mid w \in\langle b, c\rangle\right\}$ reduced words ending in $a$, and ping-pong partition $P_{\sigma}=\left\{w b^{ \pm 2} a\right\}$ and $P_{\tau}=P \backslash P_{\sigma}$. For all $N \neq 0$, we have $\sigma^{3 N}\left(P_{\tau}\right) \subseteq P_{\sigma}$ and $\tau^{3 N}\left(P_{\sigma}\right) \subseteq P_{\tau}$. Note that it is only out of an aesthetic desire to use the same power of $N$ on both generators that we use $\tau^{3}$, it is the case that $\tau^{N}\left(P_{\sigma}\right) \subseteq P_{\tau}$ for all $N \neq 0$.

Both of these examples are presented with respect to a particularly nice basis, and by taking the associated homotopy equivalence of the wedge of three circles marked by the given basis, we see that all automorphisms in the above example are upper triangular with respect to a fixed filtration. Both ping-pong arguments rely on the interaction between the suffixes in this particular upper triangular setting. This suggests a dichotomy, either length function ping-pong is possible, or every element of the group generated by a pair of Dehn twists is polynomially growing. To analyze the growth of elements in a subgroup of $\operatorname{Out}\left(F_{r}\right)$ generated by a pair of Dehn twists we will follow the cue of Bestvina, Feighn, and Handel, and understand the growth in topological models associated to the Dehn twists.

\section{Simultaneous graphs of spaces and normal forms}

Guirardel gives a topological interpretation of the intersection number of two simplicial $F_{r}$-trees. 
Theorem 5.1 [16. Theorem 7.1]. Given two non-trivial simplicial $F_{r}$ trees $A$ and $B$ there exists a cell complex $X$ with $\pi_{1}(X) \cong F_{r}$ and two 2-sided subcomplexes $Y_{A}, Y_{B} \subset X$ intersecting transversely such that $i(A, B)=\left|\pi_{0}\left(Y_{A} \cap Y_{B}\right)\right|$.

The space $X$ is constructed from the core. Let $\tilde{X}=\widehat{\mathcal{C}}(A, B) \times T$ where $T \cong \tilde{R}_{r}$ is the universal cover of a fixed wedge of $r$ circles. Let $M_{A}$ be the set of midpoints of edges of $A$ and $M_{B}$ be the set of midpoints of edges of $B$. The spaces $\tilde{Y}_{A}=\pi_{A}^{-1}\left(M_{A}\right) \times T$ and $\tilde{Y}_{B}=\pi_{B}^{-1}\left(M_{B}\right) \times T$ are a family of two-sided subcomplexes of $\tilde{X}$. The connected components of $\tilde{Y}_{A} \cap Y_{B}$ are of the form $x \times T$ where $x$ is a point in the interior of a 2-cell of $\widehat{\mathcal{C}}(A, B)$ or a midpoint of an edge in $\widehat{\mathcal{C}} \backslash \mathcal{C}$. The intersections of the form $x \times T$ when $x$ is a midpoint of an edge in the augmented core are not transverse, indeed $x \times T$ is a connected component of both $\tilde{Y}_{A}$ and $\tilde{Y}_{B}$ in this case. A transverse intersection can be obtained by instead using $M_{B}^{\prime}$ and an equivariant choice of points in the interior of the edges of $B$ none of which are the midpoints, denote this perturbation of $\tilde{Y}_{B}$ by $\tilde{Y}_{B}^{\prime}$. The connected components of $\tilde{Y}_{A} \cap \tilde{Y}_{B}^{\prime}$ are in one-to-one correspondence with the 2-cells of $\widehat{\mathcal{C}}(A, B)$. The quotients by the diagonal $F_{r}$ action, denoted $X, Y_{A}$, and $Y_{B}^{\prime}$ respectively, are the desired spaces.

These quotient spaces can be viewed through the lens of model spaces for graphs of groups, discussed in Section 2.2. Let $\bar{A}$ and $\bar{B}$ be the graphs of groups covered by $A$ and $B$ respectively. The compositions $\pi_{A} \circ \pi_{\widehat{\mathcal{C}}}$ and $\pi_{B} \circ \pi_{\widehat{\mathcal{C}}}$ of projection maps descend to the quotient and give maps $q_{A}: X \rightarrow \bar{A}$ and $q_{B}: X \rightarrow \bar{B}$. These maps make $X$ a graph of spaces over $\bar{A}$ and $\bar{B}$ simultaneously, with the connected components of $Y_{A}$ and $Y_{B}$ in the role of edge spaces. Denote by $\mathcal{A}$ and $\mathcal{B}$ the graphs of spaces structures on $X$ induced by $q_{A}$ and $q_{B}$ respectively, with $\mathcal{A}_{v}=q_{A}^{-1}(v)$ the vertex space over $v \in V(\bar{A}), \mathcal{A}_{e}=q^{-1}(e)$ the mapping cylinder over the midpoint space $\mathcal{A}_{e}^{m}=q^{-1}\left(m_{e}\right)$ of an edge $e \in E(\bar{A})$, and similar notation for $\mathcal{B}$. The goal of this section is to establish a normal form for paths and circuits in a simultaneous graph of spaces. This behavior of the core is captured in the following definition.

Definition 5.2. Let $\bar{A}$ and $\bar{B}$ be two $F_{r}$ graphs of groups. A complex $X$ is a simultaneous graph of spaces resolving $\bar{A}$ and $\bar{B}$ if there are maps $q_{A}: X \rightarrow \bar{A}$ and $q_{B}: X \rightarrow \bar{B}$ making $X$ a graph of spaces for $\bar{A}$ and $\bar{B}$ respectively (the induced structures denoted $\mathcal{A}$ and $\mathcal{B}$ ), and the following conditions on subspaces are satisfied:

(i) The midpoint spaces $\mathcal{A}_{e}^{m}$ and $\mathcal{B}_{f}^{m}$ are either equal or intersect transversely for all edges $e \in E(\bar{A})$ and $f \in E(\bar{B})$.

(ii) The intersection $\mathcal{A}_{v} \cap \mathcal{B}_{e}$ is the mapping cylinder for the maps of $\mathcal{A}_{v} \cap \mathcal{B}_{e}^{m}$ into $\mathcal{A}_{v} \cap$ $\mathcal{B}_{o(e)}$ and $\mathcal{A}_{v} \cap \mathcal{B}_{t(e)}$ as a sub-mapping cylinder of $\mathcal{B}_{e}$.

The core of $X$ is the subcomplex

$$
\bigcup_{\substack{e \in E(\bar{A}) \\ f \in E(\bar{B})}} \mathcal{A}_{e} \cap \mathcal{B}_{f}
$$

A subcomplex $Y=\mathcal{A}_{e} \cap \mathcal{B}_{f}$ of the core is twice-light if $\mathcal{A}_{e}^{m}=\mathcal{B}_{f}^{m}$.

Corollary 5.3. For any two $F_{r}$ graphs of groups $\bar{A}$ and $\bar{B}$ there is a simultaneous graph of spaces resolving them.

Proof. The space $X$ constructed in the proof of Theorem 5.1 from the core of the Bass-Serre trees covering $\bar{A}$ and $\bar{B}$ is the desired space. 
REMARK 5.4. When $X=\widehat{\mathcal{C}} \times_{F_{r}} T$, the core of $X$ is the closure of the preimages of the interiors of the 2-cells of $\widehat{\mathcal{C}}$ and the edges of $\widehat{\mathcal{C}} \backslash \mathcal{C}$. The latter are the twice-light subcomplexes.

Edges $e \subseteq X^{(1)}$ in the 1-skeleton of a simultaneous graph of spaces fall into a taxonomy given by the two decompositions. Recall that in a single graph of spaces structure $\mathcal{X}$, an edge in $X^{(1)}$ is $\mathcal{X}$-nodal if it lies in a vertex space, and $\mathcal{X}$-crossing otherwise. We extend this terminology to a simultaneous graph of spaces.

Definition 5.5. Let $e \subseteq X^{(1)}$ be an edge in the 1-skeleton of a simultaneous graph of spaces resolving $\bar{A}$ and $\bar{B}$. We say $e$ is

nodal if it is both $\mathcal{A}$ - and $\mathcal{B}$-nodal,

$\mathcal{A}$-crossing if it is $\mathcal{A}$-crossing but $\mathcal{B}$-nodal,

$\mathcal{B}$-crossing if it is $\mathcal{B}$-crossing but $\mathcal{A}$-nodal,

double-crossing if it is both $\mathcal{A}$-crossing and $\mathcal{B}$-crossing.

The possible ambiguity of terminology will be avoided by always making clear whether we are considering a single graph of spaces structure or a simultaneous graph of spaces structure.

For a single graph of spaces, based paths have a normal form that gives a topological counterpart to the Bass-Serre normal form for the fundamental groupoid. Recall Lemma 2.15, that every path based in the one skeleton of a graph of spaces is homotopic relative to the endpoints to a path

$$
v_{0} H_{1} v_{1} H_{2} \cdots H_{n} v_{n}
$$

where each $v_{i}$ is a (possibly trivial) tight edge path of $\mathcal{X}$-nodal edges, each $H_{i}$ is $\mathcal{X}$-crossing, and for all $1 \leq i \leq n-1, H_{i} v_{i} H_{i+1}$ is not homotopic relative to the endpoints to an $\mathcal{X}$-nodal edge path. A similar normal form is possible in a simultaneous graph of spaces.

Lemma 5.6. Every path in $X$, a simultaneous graph of spaces resolving $\bar{A}$ and $\bar{B}$, is homotopic relative to the endpoints to a path of the form (called simultaneous normal form)

$$
W_{0,0} K_{0,1} W_{0,1} \cdots K_{0, n_{0}} W_{0, n_{0}} H_{1} W_{1,0} \cdots H_{m} W_{m, 0} K_{m, 1} \cdots K_{m, n_{m}} W_{m, n_{m}}
$$

where the $W_{i, j}$ are (possibly trivial) tight edge paths of nodal edges, the $K_{i, j}$ are $\mathcal{B}$-crossing edges, and the $H_{i}$ are either $\mathcal{A}$-crossing or double-crossing edges. Further this path is in normal form for both $\mathcal{A}$ and $\mathcal{B}$, so that the number of $\mathcal{B}$-crossing edges plus double-crossing edges and the number of $\mathcal{A}$-crossing edges plus double-crossing edges are both invariants of the relative homotopy class of the path. A similar statement holds for free homotopy classes of loops.

Proof. Throughout this proof all homotopies will be homotopies of paths relative to the endpoints. Suppose $\gamma$ is a path in $X$. First, by Lemma 2.15, $\gamma$ is homotopic to a path in $\mathcal{A}$-normal form

$$
v_{0} H_{1} v_{1} H_{2} v_{2} \cdots H_{m} v_{m}
$$

with each $v_{i}$ an $\mathcal{A}$-nodal path and each $H_{i}$ either $\mathcal{A}$-crossing or double-crossing. With respect to $\mathcal{B}$, each $v_{i}$ is an edge path, not necessarily in normal form, of the form

$$
W_{i, 0} K_{i, 1} W_{i, 1} \cdots K_{i, n_{i}} W_{i, n_{i}}
$$

where each $W_{i, j}$ is $\mathcal{B}$-nodal (and so nodal in the simultaneous graph of spaces) and each $K_{i, j}$ is $\mathcal{B}$-crossing (in the simultaneous graph of spaces sense). We can take this path to $\mathcal{B}$-normal 
form by erasing pairs of crossing edges, but we must do so without introducing $\mathcal{A}$-crossing edges.

Suppose for some $i$ the path $K_{i, j} W_{i, j} K_{i, j+1}$ is homotopic to a path $W_{i j}^{\prime}$ that is $\mathcal{B}$-nodal. Suppress the common index $i$. Let $p$ be the vertex of $\bar{A}$ such that $K_{j} W_{j} K_{j+1} \subseteq \mathcal{A}_{p}, e$ the edge of $\bar{B}$ such that $K_{j} W_{j} K_{j+1} \subseteq \mathcal{B}_{e}$, so that $W_{j} \subseteq \mathcal{B}_{t(e)}$ and $W_{j}^{\prime} \subseteq \mathcal{B}_{o(e)}$. Since $K_{j} W_{j} K_{j+1} \subseteq \mathcal{A}_{p} \cap \mathcal{B}_{e}$, this is a path in the mapping cylinder for the inclusions of $\mathcal{A}_{p} \cap \mathcal{B}_{e}^{m}$ into the endpoints, and $W_{j}$ is a fiber of this cylinder. Thus $K_{j} W_{j} K_{j+1}$ is homotopic via a homotopy in $\mathcal{A}_{p} \cap \mathcal{B}_{e}$ to a path $W_{j}^{\prime \prime} \subseteq \mathcal{A}_{p} \cap \mathcal{B}_{o(e)}$. Using $W_{j}^{\prime \prime}$ to erase the pair of crossing edges, we see that each $v_{i}$ can be expressed in $\mathcal{B}$ normal form and remain $\mathcal{A}$-nodal. Thus $\gamma$ is homotopic to a path of the form

$$
W_{0,0} K_{0,1} W_{0,1} \cdots K_{0, n_{0}} W_{0, n_{0}} H_{1} W_{1,0} \cdots H_{m} W_{m, 0} K_{m, 1} \cdots K_{m, n_{m}} W_{m, n_{m}} .
$$

This path may not be in $\mathcal{B}$-normal form. There are two possible cases, and in both we will show that it is possible to erase a pair of $\mathcal{B}$-crossing edges without destroying $\mathcal{A}$-normal form.

First, suppose this path is not $\mathcal{B}$-normal because there is some $i$ such that $K_{i, n_{i}} W_{i, n_{i}} H_{i+1}$ (or symmetrically $H_{i} W_{i, 0} K_{i, 1}$ ) is homotopic to a path $W_{i}^{\prime}$ that is $\mathcal{B}$-nodal. Let $f$ be the edge of $\bar{B}$ crossed by $K_{i, n_{i}}$. In this case, the endpoints map to $o(f)$ by $q_{B}$, and $q_{B}\left(W_{i, 0}\right)=t(f)$ so by continuity $q_{B}\left(H_{i+1}\right)=\bar{f}$; thus $H_{i+1}$ is double-crossing. Note that this path is already in $\mathcal{A}$-normal form. Again suppress the common index, and take $K_{n} W_{n} H$ to a path in the $\mathcal{B}$-vertex space $\mathcal{B}_{o(f)}$. This path will have some number of $\mathcal{A}$-crossing edges, but similar to the previous paragraph, this path is homotopic to one in $\mathcal{A}$-normal form via a homotopy inside $\mathcal{B}_{o(f)}$, so that by Lemma 2.15 $K_{n} W_{n} H$ is homotopic to a path of the form $W_{n}^{\prime} H^{\prime} W^{\prime}$ with exactly one $\mathcal{A}$-crossing edge, and $W_{n}^{\prime}$ and $W^{\prime}$ are nodal.

Second, suppose the resulting path is not $\mathcal{B}$-normal because there is some $i$ such that $K_{i, n_{i}} W_{i, n_{i}} H_{i+1} W_{i+1,0} K_{i, 0}$ is homotopic to a path $W_{i}^{\prime}$ that is $\mathcal{B}$-nodal, contained in the vertex space of $q \in V(\bar{B})$. In this case $H_{i+1}$ must be $\mathcal{A}$-crossing. As before, the path $W_{i}^{\prime} \subseteq \mathcal{B}_{q}$ is homotopic to a path in $\mathcal{A}$-normal form contained in $\mathcal{B}_{q}$.

In both cases, the number of $\mathcal{A}$-crossing edges is maintained, so the result is in $\mathcal{A}$-normal form.

Therefore, a path $\gamma$ is homotopic to a path in simultaneous normal form, and can be taken to this normal form by composing the following homotopies:

(i) take $\gamma$ to $\mathcal{A}$-normal form,

(ii) take each $\mathcal{A}$-nodal sub-path to $\mathcal{B}$-normal form within the appropriate $\mathcal{A}$ vertex space,

(iii) erase remaining pairs of $\mathcal{B}$-crossing edges, maintaining $\mathcal{A}$-normal form.

The homotopy invariance of the number of crossing edge types follows immediately from Lemma 2.15.

\section{Twisting in graphs of spaces}

A Dehn twist on a graph of groups can be realized by an action on based homotopy classes of paths in a graph of spaces. Let $\Gamma$ be a graph of groups modeled by the graph of spaces $X$, and $D$ a Dehn twist based on $\Gamma$. Each crossing edge $H \in X^{(1)}$ lies over some edge $e \in E(\Gamma)$. For each crossing edge $H$ pick a loop $\gamma_{H}$ in $\mathcal{X}_{t(e)}$, contained in the image of $\mathcal{X}_{e} \times\{1\}$ representing $z_{e}$ and based at $t(H)$. The action of $D$ on a crossing edge is the concatenation

$$
D(H)=H \gamma_{H}
$$

The action is extended to an action on all paths in $X^{(1)}$ by concatenation and $D(v)=v$ for every nodal path, and to based homotopy classes by taking one-skeleton representatives. That this action is well-defined and represents the Dehn twist $D$ faithfully follows from noting that 
the below diagram of fundamental groupoids commutes.

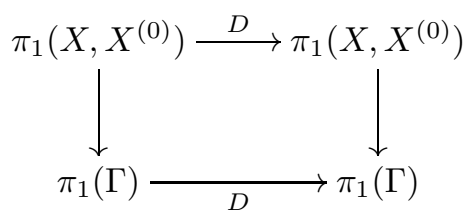

Also from this diagram we see that if a path $\gamma$ is in normal form, then so is $D(\gamma)$, with the same crossing edges.

Extending this to the setting of a simultaneous graph of spaces resolving $\bar{A}$ and $\bar{B}$, and twists $\tilde{\sigma}$ based on $\bar{A}$ and $\tilde{\tau}$ based on $\bar{B}$, we see that $\tilde{\sigma}$ preserves $\mathcal{A}$-normal form (though we can make no comment on the $\mathcal{B}$-normal form) and a symmetric statement holds for $\tilde{\tau}$. To understand the behavior of paths in simultaneous normal form we must track the extent to which $\tilde{\sigma}$ alters the number of $\mathcal{B}$-crossing edges and vise-versa. This interaction is contained entirely in the graphs of groups, and applies to all twists based on the graphs.

Definition 6.1. The edge twist digraph $\mathcal{E} \mathcal{T}(\bar{A}, \bar{B})$ of two small graphs of groups is a directed graph with vertex set

$$
V(\mathcal{E} \mathcal{T})=\{(e, \bar{e}), \mid e \in E(\bar{A})\} \cup\{(f, \bar{f}) \mid f \in E(\bar{B})\},
$$

directed edges $((e, \bar{e}),(f, \bar{f})) e \in E(\bar{A}), f \in E(\bar{B})$ when a generator $\bar{A}_{e}=\left\langle z_{e}\right\rangle$ or its inverse uses $f$ or $\bar{f}$ in cyclically reduced normal form with respect to $\bar{B}$, and directed edges $((f, \bar{f}),(e, \bar{e}))$ $f \in E(\bar{B}), e \in E(\bar{A})$ when a generator $\bar{B}_{f}=\left\langle z_{f}\right\rangle$ or its inverse uses $e$ or $\bar{e}$ in cyclically reduced normal form with respect to $\bar{A}$.

EXAMPLE 6.2. Suppose $\bar{A}$ and $\bar{B}$ are hyperbolic-hyperbolic one-edge graphs of groups with edges $a$ and $b$ respectively. Then $\mathcal{E} \mathcal{T}(\bar{A}, \bar{B})$ is the following digraph.

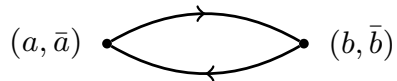

Example 6.3. Let $\bar{A}, \bar{B}$, and $\bar{C}$ be the graphs of groups from Examples 4.13 and 4.14 , Their edge twist digraphs are the following. Both $\mathcal{E} \mathcal{T}(\bar{A}, \bar{C})$ and $\mathcal{E} \mathcal{T}(\bar{B}, \bar{C})$ have two verticies and no edges. The digraph $\mathcal{E} \mathcal{T}(\bar{A}, \bar{B})$ has a single directed edge from $(a, \bar{a})$ to $(b, \bar{b})$.

This definition is made somewhat cumbersome by the presence of orientation. The vertex set is the unoriented edges of the two graphs of groups, and the property of crossing an unoriented edge in normal form is shared by the generator and its inverse. We encapsulate the resulting awkwardness here, so that subsequent arguments about paths in simultaneous normal form are clear.

The edge-twist structure controls the growth rate of elements in any group generated by a twist $\tilde{\sigma}$ on $\bar{A}$ and $\tilde{\tau}$ on $\bar{B}$.

Lemma 6.4. Suppose $\bar{A}$ and $\bar{B}$ are minimal visible small graphs of groups with free fundamental group $F_{r}$ and $\mathcal{E} \mathcal{T}(\bar{A}, \bar{B})$ is acyclic. Then for any pair of Dehn twists $\sigma, \tau \in \operatorname{Out}\left(F_{r}\right)$ represented by $\tilde{\sigma}$ based on $\bar{A}$ and $\tilde{\tau}$ based on $\bar{B}$, every element of $\langle\sigma, \tau\rangle \leq \operatorname{Out}\left(F_{r}\right)$ is polynomially growing. Moreover, the growth degree is at most the length of the longest directed path in $\mathcal{E} \mathcal{T}(\bar{A}, \bar{B})$. 
Proof. Let $X=\widehat{\mathcal{C}}(A, B) \times_{F_{r}} T$ be the simultaneous graph of spaces constructed from the augmented core of the Bass-Serre trees $A$ and $B$ for $\bar{A}$ and $\bar{B}$, with $\bar{T}$ a wedge of circles, equipped with the $\ell_{1}$ metric. Note that $\tilde{X}$ has an equivariant Lipschitz surjection to $T$ given by projection and that this descends to a Lipschitz homotopy equivalence on the quotient, denoted $\rho: X \rightarrow \bar{T}$. Further, if $\gamma$ is a loop in $X^{(1)}$ representing a conjugacy class $[g]$ of $\pi_{1}(\bar{T})$,

$$
\ell_{T}(g) \leq|\rho(\gamma)|_{\bar{T}} \leq \operatorname{Lip}(\rho) \cdot|\gamma|_{X}
$$

where $|\cdot|$ is the arclength. Further, for any $w \in\langle\sigma, \tau\rangle$, by expressing $w$ as a word in the generators we get an action on paths $\tilde{w}$, with the property that $w(g)$ is represented by $\tilde{w}(\gamma)$.

Therefore, it suffices to give a polynomial bound on the growth of paths in $X$ under the topological representatives of $\sigma$ and $\tau$. Moreover, for any edge path $\gamma$ the growth under the action of $\tilde{\sigma}$ and $\tilde{\tau}$ is bounded by the number of $\mathcal{A}$-crossing edges of $\gamma$ times the growth of $\mathcal{A}$-crossing edges plus the similar quantity for $\mathcal{B}$-crossing edges. So it suffices to bound the growth of crossing edges. (Note, this is an upper bound, we make no attempt to understand cancellation that might happen, as a result these bounds could be quite bad.)

First, as a technical convenience, replace $\bar{A}$ and $\bar{B}$ by the isomorphic graphs of groups constructed from $A$ and $B$ using a fundamental domain in each that is the image under projection of a fundamental domain for $\widehat{\mathcal{C}}(A, B)$, so that the edge groups of edges in each of $\bar{A}$ and $\bar{B}$ whose orbits are covered by diagonals of the core are not just conjugate, but equal on the nose. This does not change the outer automorphism class of the Dehn twists under consideration, nor does it change the edge twist graph.

Suppose $D$ is a double-crossing edge of $X^{(1)}$ lying over $e \in E(A)$ and $f \in E(B)$, so that the edge group $\bar{A}_{e}=\bar{B}_{f}=\langle z\rangle$ with common generator $z$. The cyclically reduced normal form of $z$ with respect to $\bar{A}$ based at $t(e)$ is $\iota_{e}(z)$; and with respect to $\bar{B}$ based at $t(f)$ is $\iota_{f}(z)$. Since both of these normal forms for $z$ contain no edges, the vertices $(e, \bar{e})$ and $(f, \bar{f})$ of $\mathcal{E} \mathcal{T}(\bar{A}, \bar{B})$ have no outgoing edges. Moreover, we can choose a loop representing a generator $z$ that is nodal and based at $t(D)$, and alter the topological representatives of $\tilde{\sigma}$ and $\tilde{\tau}$ so that $\tilde{\sigma}(D)=\gamma^{a}$ and $\tilde{\tau}(D)=\gamma^{b}$, concatenations of either $\gamma$ or its reverse, according to the expression of the twisters of $\tilde{\sigma}$ about $e$ and $\tilde{\tau}$ about $f$ in terms of the generator $z$. Thus,

$$
\tilde{\sigma}^{s_{n}} \tilde{\tau}^{t_{n}} \cdots \tilde{\sigma}_{1}^{s} \tilde{\tau}^{t_{1}}(D)=D \gamma^{a \sum s_{i}+b \sum t_{i}}
$$

which has edge length at most linear in $\sum\left|s_{i}\right|+\sum\left|t_{i}\right|$.

Suppose $H$ is an $\mathcal{A}$ - or $\mathcal{B}$-crossing edge of $X^{(1)}$ lying over $(e, \bar{e}) \in V(\mathcal{E} \mathcal{T})$. Let $d_{e}$ be the length of the longest directed path in $\mathcal{E} \mathcal{T}(\bar{A}, \bar{B})$ starting at $e$. We will use the notation $\operatorname{poly}_{d}(x)$ to stand for some polynomial of degree $d$ in $x$, as we are looking for an upper bound and making no attempt to estimate coefficients.

Claim. For any crossing edge $H$, the length of $\tilde{\sigma}^{s_{n}} \tilde{\tau}^{s_{n}} \cdots \tilde{\sigma}^{s_{1}} \tilde{\tau}^{t_{1}}(H)$ is at most $\operatorname{poly}_{d_{e}+1}\left(\sum\left|s_{i}\right|+\sum\left|t_{i}\right|\right)$.

Proof. For double-crossing edges, the argument in the previous discussion establishes this claim. It remains to establish the claim for edges that are crossing but not double-crossing. The proof is by induction on $d_{e}$. As the argument is symmetric, we will suppose $H$ is $\mathcal{A}$-crossing, so that $e \in E(A)$.

Base Case: $d_{e}=0$. Let $\gamma_{H}$ be a loop representing a generator $z_{e}$ of $\bar{A}_{e}$ based at $t(H)$ and in simultaneous normal form. Since $(e, \bar{e})$ has no outgoing edges in $\mathcal{E} \mathcal{T}$, the loop $\gamma_{H}$ is $\mathcal{B}$-nodal. Let $a$ be the power so that $z_{e}^{a}$ is the $e$ twister of $\tilde{\sigma}$. Use $\gamma_{e}^{a}$ in the topological representative of $\tilde{\sigma}$. Then for any $s, \tilde{\sigma}^{s}(H)=H \gamma_{e}^{a s}$ is a $\mathcal{B}$-nodal path, and we have

$$
\tilde{\sigma}^{s_{n}} \tilde{\tau}^{t_{n}} \cdots \tilde{\sigma}^{s_{1}} \tilde{\tau}^{t_{1}}(H)=H \gamma_{H}^{a \sum s_{i}}
$$

which has edge length at most linear in $\sum\left|s_{i}\right|+\sum\left|t_{i}\right|$, as required. 
Inductive Step: $d_{e}>0$. Since $d_{e}>0,(e, \bar{e})$ has neighbors $\left(f_{1}, \bar{f}_{1}\right), \ldots,\left(f_{k}, \bar{f}_{k}\right)$. As before, use a simultaneous normal form representative $\gamma_{H}$ for a generator $z_{e}$ of $\bar{A}_{e}$ based at $t(H)$, so that $\sigma(H)=\gamma_{H}^{a}$. Since $\gamma_{H}$ has an $\mathcal{A}$-nodal representative by definition, we have in simultaneous normal form

$$
\gamma_{H}=W_{0} K_{1} \cdots K_{m} W_{m}
$$

where $K_{i}$ lies over either $f_{k_{i}}$ or $\bar{f}_{k_{i}}$ by the definition of the edge twist graph. Further, for each $f_{i}$, the longest path in $\mathcal{E} \mathcal{T}$ based at $f_{i}$, has length at most $d_{e}-1$. Calculating, we have

$$
\begin{gathered}
\tilde{\sigma}^{s_{n}} \tilde{\tau}^{t_{n}} \cdots \tilde{\sigma}^{s_{1}} \tilde{\tau}^{t_{1}}(H)=H \gamma_{H}^{a s_{n}} \tilde{\sigma}^{s_{n}} \tilde{\tau}^{t_{n}}\left(\gamma_{H}^{a s_{n-1}}\right) \\
\cdot \tilde{\sigma}^{s_{n}} \tilde{\tau}^{t_{n}} \tilde{\sigma}^{s_{n-1}} \tilde{\tau}^{t_{n-1}}\left(\gamma_{H}^{a s_{n-2}}\right) \\
\vdots \\
\cdot \tilde{\sigma}^{s_{n}} \tilde{\tau}^{t_{n}} \ldots \tilde{\tau}^{t_{2}}\left(\gamma_{H}^{a s_{1}}\right) .
\end{gathered}
$$

By the induction hypothesis, the length of each $K_{v_{i}}$ under a composition of powers of $\tilde{\sigma}$ and $\tilde{\tau}$ is bounded by a polynomial of degree at most $d_{e}$. Hence the path $\tilde{\sigma}^{s_{n}} \tilde{\tau}^{t_{n}} \cdots \tilde{\sigma}^{s_{2}} \tilde{\tau}^{t_{2}}\left(\gamma^{a s_{1}}\right)$ has length at most

$$
\left|a s_{1}\right| \cdot \operatorname{poly}_{d_{e}}\left(\sum_{i \geq 2}\left|s_{i}\right|+\left|t_{i}\right|\right) .
$$

Similarly, we bound the lengths of the other components and estimate

$$
\begin{aligned}
\left|\tilde{\sigma}^{s_{n}} \tilde{\tau}^{t_{n}} \cdots \tilde{\sigma}^{s_{1}} \tilde{\tau}^{t_{1}}(H)\right| & \leq\left|H \gamma_{H}^{a s_{n}} \tilde{\sigma}^{s_{n}} \tilde{\tau}^{t_{n}}\left(\gamma_{H}^{a s_{n-1}}\right)\right| \\
& +\left|\tilde{\sigma}^{s_{n}} \tilde{\tau}^{t_{n}} \tilde{\sigma}^{s_{n-1}} \tilde{\tau}^{t_{n-1}}\left(\gamma_{H}^{a s_{n-2}}\right)\right| \\
& \vdots \\
& +\left|\tilde{\sigma}^{s_{n}} \tilde{\tau}^{t_{n}} \cdots \tilde{\tau}^{t_{2}}\left(\gamma_{H}^{a s_{1}}\right)\right| \\
& \leq \sum_{i=1}^{n}\left|a s_{i}\right| \cdot \operatorname{poly}_{d_{e}}\left(\sum_{j>i}\left|s_{j}\right|+\left|t_{j}\right|\right)
\end{aligned}
$$

and this quantity is in turn at most poly $d_{e}+1\left(\sum\left|s_{i}\right|+\left|t_{i}\right|\right)$. This completes the claim.

Finally, suppose $w=\sigma^{s_{n}} \tau^{s_{n}} \cdots \sigma^{s_{1}} \tau^{t_{1}} \in\langle\sigma, \tau\rangle$. For any $g \in F$, let $\gamma$ be a loop in simultaneous normal form representing the conjugacy class of $g$ in $X^{(1)}$. The length $\ell_{T}\left(w^{N}(g)\right)$ is bounded by the length in $X$ of $\tilde{w}^{N}(\gamma)$, which by the claim is at most

$$
\operatorname{poly}_{d+1}\left(N \cdot\left(\sum\left|s_{i}\right|+\left|t_{i}\right|\right)\right)
$$

where $d$ is the length of the longest directed path in $\mathcal{E} \mathcal{T}$. This is a polynomial of degree $d+1$ in $N$, which completes the lemma.

An interesting question, which we do not pursue here is whether or not Lemma 6.4 is sharp. That is, if $\mathcal{E} \mathcal{T}(\bar{A}, \bar{B})$ contains a cycle, is there some pair of twists $\sigma, \tau$ with representatives based on $\bar{A}$ and $\bar{B}$ respectively so that the group generated contains an outer automorphism with an exponentially growing stratum? In the setting of one-edge splittings, Clay and Pettet's result is in this direction: two one-edge graphs of groups that fill have a directed cycle of length two in their edge-twist graphs; the group generated contains a fully irreducible element, which is exponentially growing. 


\section{Dehn twists on incompatible graphs generate free groups}

We are now in a position to give a full converse to Lemma 4.1. The proof is by two cases, decided by the structure of the edge-twist graph. When the edge-twist graph contains a cycle, this cycle enables a length function ping-pong argument that is almost identical to the proof of Lemma 4.11. When the edge-twist graph is acyclic, the group generated by the pair of twists is polynomially growing and we analyze its structure using the Kolchin theorem for $\operatorname{Out}\left(F_{r}\right)$ of Bestvina, Feighn, and Handel. As the two arguments are significantly different, we present them as two lemmas.

Lemma 7.1. Suppose $\sigma$ and $\tau$ are Dehn twists of $F_{r}$ with efficient representatives $\tilde{\sigma}$ and $\tilde{\tau}$ on graphs of groups $\bar{A}$ and $\bar{B}$ such that $\mathcal{E} \mathcal{T}(\bar{A}, \bar{B})$ contains a cycle. Then, for all $n \geq N=$ $48 r^{2}-48 r+3$, the group $\left\langle\sigma^{N}, \tau^{N}\right\rangle \cong F_{2}$.

Proof. Let $\left(u_{1}, \bar{u}_{1}\right), \ldots,\left(u_{c}, \bar{u}_{c}\right)$ and $\left(v_{1}, \bar{v}_{1}\right), \ldots,\left(v_{c}, \bar{v}_{c}\right)$ be the vertices of a primitive cycle in $\mathcal{E} \mathcal{T}$, with $u_{i} \in E(\bar{A})$ and $v_{i} \in E(\bar{B})$. (It is psychologically unfortunate to use $u$ and $v$ for edges, but this usage is only for this proof.) The index $c$ is the same for both sets as $\mathcal{E} \mathcal{T}$ is bipartite, and no vertex $\left(u_{i}, \bar{u}_{i}\right)$ or $\left(v_{i}, \bar{v}_{i}\right)$ is repeated. For each edge $u_{i}$ fix a generator $\left\langle a_{u_{i}}\right\rangle=\bar{A}_{u_{i}}$ and $s_{u_{i}} \neq 0$ so that the twister of $\tilde{\sigma}$ about $u_{i}$ is $z_{u_{i}}=a_{u_{i}}^{s_{u_{i}}}$, and similarly fix $\left\langle b_{v_{i}}\right\rangle=\bar{B}_{v_{i}}$ and $t_{i} \neq 0$. (The $s_{u}$ and $t_{v}$ are nonzero as both $\tilde{\sigma}$ and $\tilde{\tau}$ twist on every edge of their respective graphs.) Let $\bar{A}^{\prime}$ and $\bar{B}^{\prime}$ be the quotient graphs of groups obtained by collapsing $E(\bar{A}) \backslash\left\{u_{i}, \bar{u}_{i}\right\}$ and $E(\bar{B}) \backslash\left\{v_{i}, \bar{v}_{i}\right\}$ in $\bar{A}$ and $\bar{B}$ respectively.

We will again use conjugacy class ping-pong. Define a set $P$ by the partition $P=P_{\sigma} \sqcup P_{\tau}$ where

$$
\begin{aligned}
& P_{\sigma}=\left\{[w] \in P \mid \ell_{A^{\prime}}(w)<\ell_{B^{\prime}}(w)\right\} \\
& P_{\tau}=\left\{[w] \in P \mid \ell_{B^{\prime}}(w)<\ell_{A^{\prime}}(w)\right\} .
\end{aligned}
$$

This partition is non-trivial, the edge-group generators $a_{u} \in P_{\tau}$ and $b_{v} \in P_{\sigma}$.

Once more we will find an $N$ so that for all $n \geq N, \sigma^{ \pm n}\left(P_{\tau}\right) \subseteq P_{\sigma}$ and $\tau^{ \pm n}\left(P_{\sigma}\right) \subseteq P_{\tau}$, to conclude, by the ping-pong lemma, $\left\langle\sigma^{n}, \tau^{n}\right\rangle \cong F_{2}$. The argument will be symmetric, and almost identical to that of Lemma 4.11.

Suppose $[w] \in P_{\tau}$, so that $0<\ell_{A^{\prime}}(w)$. Fix a cyclically reduced representative of $w$ in transverse Bass-Serre normal form with respect to a fixed basis $\Lambda$ and $\bar{A}^{\prime}$,

$$
w=e_{1} a_{e_{1}}^{k_{1}} w_{1} e_{2} a_{e_{2}}^{k_{2}} w_{2} \cdots e_{\ell} a_{e_{\ell}}^{k_{\ell}} w_{\ell}
$$

where we are suppressing the different edge morphisms, using $\ell=\ell_{A^{\prime}}(w)$ for legibility, $e_{i} \in$ $\left\{u_{i}, \bar{u}_{i}\right\}$, and each $w_{i}$ is in the right transversal of the image of $a_{e_{i}}$ in the vertex group involved. Let $C$ be the bounded cancellation constant for the fixed basis of $F_{r}$ basis into $B^{\prime}$. With respect to this basis, after an appropriate conjugation we have the cyclically reduced conjugacy class representative $w^{\prime}$ satisfying

$$
\left|w^{\prime}\right|=\left|a_{e_{1}}^{k_{1}^{\prime}}\right|+\cdots+\left|w_{\ell-1}^{\prime}\right|+\left|a_{e_{\ell}}^{k_{\ell}^{\prime}}\right|+\left|w_{\ell}^{\prime}\right|
$$

where $w_{i}^{\prime}$ is the reduced word for the group element obtained from the arrow $a_{e_{i}}^{ \pm 1} w_{i} e_{i+1} a_{e_{i+1}}^{ \pm 1}$ after collapsing a maximal tree, and $k_{i}^{\prime}$ differs from $k_{i}$ by a fixed amount depending only on the edges, as each $w_{i}^{\prime}$ might might disturb a fixed number of adjacent copies of conjugates of $a_{i}$ depending on the particular spelling (This decomposition of $w^{\prime}$ as a reduced word with respect to $\Lambda$ follows from normal form as in the proof of Lemma 4.11) Let $\alpha=\min _{i}\left\{\ell_{B^{\prime}}\left(a_{u_{i}}\right)\right\}$. Since each $\left(u_{i}, \bar{u}_{i}\right)$ is joined to some $\left(v_{i}, \bar{v}_{i}\right)$ by an edge in $\mathcal{E} \mathcal{T}$ as they are all vertices of a cycle, $\alpha>0$. 
We have, by bounded cancellation,

$$
\begin{aligned}
\ell_{A^{\prime}}(w)>\ell_{B^{\prime}} & \geq \sum_{i=1}^{\ell}\left|k_{i}\right| \ell_{B^{\prime}}\left(a_{e_{i}}\right)-2 C\left(\Lambda, B^{\prime}\right) \ell_{A^{\prime}}(w) \\
& \geq\left(\sum_{i=1}^{p}\left|k_{i}^{\prime}\right|\right) \alpha-2 C\left(\Lambda, B^{\prime}\right) \ell_{A^{\prime}}(w) .
\end{aligned}
$$

We conclude

$$
\sum\left|k_{i}^{\prime}\right|<\left(\frac{1+2 C\left(\Lambda, B^{\prime}\right)}{\alpha}\right) \ell_{A^{\prime}}(w)
$$

Calculating with the induced action of $\tilde{\sigma}$ on arrows in $\pi_{1}\left(\bar{A}^{\prime}\right)$ and abusing notation to also call this action $\tilde{\sigma}$, we have

$$
\tilde{\sigma}^{n}(w)=e_{1} a_{e_{1}}^{s_{e_{1}} n} a_{e_{1}}^{k_{1}} \tilde{\sigma}^{n}\left(w_{1}\right) e_{2} a_{e_{2}}^{s_{e_{2}} n} a_{e_{2}}^{k_{2}} \cdots e_{\ell} a_{e_{\ell}}^{s_{e_{\ell}} n} a_{e_{\ell}}^{k_{\ell}} \tilde{\sigma}^{n}\left(w_{\ell}\right) .
$$

The possibility that $\tilde{\sigma}^{n}\left(w_{i}\right)$ is of the form $a_{e_{i}}^{\epsilon n} x_{i} a_{e_{i+1}}^{\delta n}$ is ruled out by the no positive bonding condition of the efficient representative: $\epsilon n$ and $s_{e_{i}} n$ must have the same sign, and also $\delta n$ and $s_{e_{i+1}} n$. So, reducing and applying bounded cancellation in the same fashion, we have, with $s=\min _{i}\left\{\left|s_{i}\right|\right\}$

$$
\begin{aligned}
\ell_{B^{\prime}}\left(\sigma^{n}(w)\right) & \geq \sum_{i=1}^{\ell}\left|s_{e_{i}} n+k_{i}^{\prime}\right| \ell_{B^{\prime}}\left(a_{e_{i}}\right)-2 C\left(\Lambda, B^{\prime}\right) \ell_{A^{\prime}}(w) \\
& \geq\left(|s n| \ell_{A^{\prime}}(w)-\sum_{i=1}^{p}\left|k_{i}^{\prime}\right|\right) \alpha-2 C\left(\Lambda, B^{\prime}\right) \ell_{A^{\prime}}(w) \\
& \geq\left(|s n|-\frac{1+2 C\left(\Lambda, B^{\prime}\right)}{\alpha}\right) \alpha \ell_{A^{\prime}}(w)-2 C\left(\Lambda, B^{\prime}\right) \ell_{A^{\prime}}(w)
\end{aligned}
$$

with the last step following from Equation $\dagger$. Thus we have

$$
\frac{\ell_{B^{\prime}}\left(\sigma^{n}(w)\right)}{\ell_{A^{\prime}}\left(\sigma^{n}(w)\right)}=\frac{\ell_{B^{\prime}}\left(\sigma^{n}(w)\right)}{\ell_{A^{\prime}}(w)} \geq\left(|s n|-\frac{1+2 C\left(\Lambda, B^{\prime}\right)}{\alpha}\right) \alpha-2 C\left(\Lambda, B^{\prime}\right) .
$$

Therefore, to ensure $\sigma^{n}(w) \in P_{\sigma}$ we require

$$
\left(|s n|-\frac{1+2 C\left(\Lambda, B^{\prime}\right)}{\alpha}\right) \alpha-2 C\left(\Lambda, B^{\prime}\right)>1
$$

that is,

$$
|n|>\frac{2+4 C\left(\Lambda, B^{\prime}\right)}{s \alpha} .
$$

As before, after choosing bases using Lemma 3.13 for both this calculation and a similar calculation involving $\tau$, we conclude that for all $n \geq N=48 r^{2}-48 r+3$ both $\sigma^{ \pm n}\left(P_{\tau}\right) \subseteq P_{\sigma}$ and $\tau^{ \pm n}\left(P_{\sigma}\right) \subseteq P_{\tau}$. Therefore the group $\left\langle\sigma^{n}, \tau^{n}\right\rangle \cong F_{2}$ by the ping-pong lemma.

The presence of a cycle in $\mathcal{E} \mathcal{T}(\bar{A}, \bar{B})$ is essential in the above proof; it guarantees there is some subset of twisters and edges where the growth of one restricted length function is linear in the value of the other restricted length function. Without a cycle, this kind of uniform control is unavailable, as illustrated by Examples 4.13 and 4.14. Fortunately, this is the exact case where the generated group is polynomially growing and the Kolchin theorem can be applied. Using the simultaneous upper triangular representatives a different form of ping-pong can be effected.

First we require a lemma relating the core of two efficient twists and the structure of their simultaneous upper triangular representatives. The contrapositive of this lemma will be used to find paths suitable for ping-pong, after applying the Kolchin theorem. 
Lemma 7.2. Suppose $\sigma$ and $\tau$ are Dehn twist outer automorphisms with upper-triangular relative train-track representatives $\hat{\sigma}$ and $\hat{\tau}$ with respect to a filtered graph $\emptyset=\Gamma_{0} \subsetneq \Gamma_{1} \subsetneq$ $\cdots \subsetneq \Gamma_{k}=\Gamma$, and efficient representatives $\tilde{\sigma}$ and $\tilde{\tau}$ on graphs of groups $\bar{A}$ and $\bar{B}$ covered by $A$ and $B$ respectively. If

(i) Every suffix of $\hat{\sigma}$ is $\hat{\tau}$-Nielsen,

(ii) Every suffix of $\hat{\tau}$ is $\hat{\sigma}$-Nielsen,

(iii) For every edge $E_{i} \in \Gamma_{i} \backslash \Gamma_{i-1}$ if $E_{i}$ is a linear edge of both $\hat{\sigma}$ and $\hat{\tau}$ the associated primitive Nielsen paths are equal (up to orientation),

then $i(A, B)=0$.

Proof. The construction of efficient representatives in Lemma 3.10 from a relative traintrack involves first folding conjugates, then a series of folding edges in linear families, and finally a series of graph of groups Stallings folds; it follows from Cohen and Lustig's parabolic orbits theorem that the simplicial structure of the resulting tree is unique (Theorem 3.8 and Corollary (3.9). We carry out the same construction, using both $\hat{\sigma}$-linear edges and $\hat{\tau}$-linear edges. A joint linear family is a collection of single edges $\left\{E_{i}\right\}$ which have either $\hat{\sigma}$ or $\hat{\tau}$ suffixes that are a power of a fixed primitive Nielsen path $\gamma$. By hypothesis, if two edges $E_{i}$ and $E_{j}$ are in the same linear family for one of the maps, then they are in the same joint linear family. As in the construction of efficient representatives, we first fold conjugates and then linear families; the hypotheses ensure that this can be done in a compatible fashion. The resulting folded graph and folded representatives, $\hat{\sigma}^{\prime}, \hat{\tau}^{\prime}: \Gamma^{\prime} \rightarrow \Gamma^{\prime}$ are still upper triangular, represent $\sigma$ and $\tau$ respectively, and have the property that every linear family contains one edge.

We now construct a tree $C$ that resolves the trees $A$ and $B$. First, recall that the efficient representative of $\hat{\sigma}^{\prime}$ on a tree $A$ can be constructed from $\Gamma^{\prime}$ as follows. Start with $A_{0}$ obtained from the universal cover of $\Gamma^{\prime}$ by collapsing all $\hat{\sigma}^{\prime}$ fixed edges of $\Gamma^{\prime}$. We then work up the remaining orbits of edges of $A_{0}$ by the filtration of $\Gamma^{\prime}$. If $\hat{\sigma}^{\prime}\left(E_{i}\right)=E_{i}$ then set $A_{i}=A_{i-1}$, otherwise $\hat{\sigma}^{\prime}\left(E_{i}\right)=E_{i} u_{i}$, and each lift of $u_{i}$ by construction represents an element in the vertex group based at a lift of $t\left(E_{i}\right)$; the tree $A_{i}$ is obtained from $A_{i-1}$ by folding the associated primitive Nielsen path $\gamma_{i}$ over $E_{i}$ (the details are in Lemma 3.10), and the result $A_{k}$ is $A$. To construct the resolving tree, we start with $C_{0}$, obtained from the universal cover of $\Gamma^{\prime}$ by collapsing all edges that are fixed by both $\hat{\sigma}^{\prime}$ and $\hat{\tau}^{\prime}$. Then, working up the hierarchy of $\Gamma$, if $E_{i}$ is both $\hat{\sigma}^{\prime}$ and $\hat{\tau}^{\prime}$ fixed, set $C_{i}=C_{i-1}$, otherwise $\hat{\sigma}^{\prime}\left(E_{i}\right)=E_{i} \gamma_{i}^{s}$ and $\hat{\tau}^{\prime}\left(E_{i}\right)=E_{i} \gamma_{i}^{t}$ for a primitive Nielsen path $\gamma_{i}$ (allowing the possibility $s$ or $t$ is zero); in this case by construction lifts of $\gamma_{i}$ represent elements in the vertex stabilizers of lifts of $t\left(E_{i}\right)$, so we obtain $C_{i}$ from $C_{i-1}$ by pulling $\gamma_{i}$ over $E_{i}$. The desired resolving tree is $C=C_{k}$. It is readily apparent from this construction that $C$ maps to $A$ and $B$ by collapse maps: collapse any remaining $\sigma$ fixed edges of $C$ to obtain $A$, and any remaining $\tau$ fixed edges of $C$ to obtain $B$.

By Theorem 2.19, since $A$ and $B$ have a common refinement, the core is a tree and therefore contains no rectangles, whence $i(A, B)=0$.

With the relationship between the core and upper triangular representatives understood, we complete the remaining parts of the proof of the main theorem.

Lemma 7.3. Suppose $\tilde{\sigma}, \tilde{\tau}$ are efficient Dehn twists with trivial image in $G L(r, \mathbb{Z} / 3 \mathbb{Z})$ based on $\bar{A}$ and $\bar{B}$ respectively. If $\mathcal{E} \mathcal{T}(\bar{A}, \bar{B})$ is acyclic and $i(A, B)>0$, then $\left\langle\sigma^{3}, \tau^{3}\right\rangle \cong F_{2}$.

For the proof we require some notation. For two paths $\gamma, \delta \subseteq \Gamma$ with the same initial point, the overlap length is defined by $\theta(\gamma, \delta)=\frac{1}{2}\left(\operatorname{length}_{\Gamma}([\gamma])+\operatorname{length}_{\Gamma}([\delta])-\operatorname{length}([\bar{\gamma} \delta])\right)$, where we use the metric on $\Gamma$ induced by assigning each edge length one. We will often understand 
the overlap length by calculating the common initial segment of two tight paths, this is the connected component of the intersection of lifts of $\gamma$ and $\delta$ based at a common point. The length of this segment is equal to the overlap length.

Proof. By Lemma 6.4 the group $\langle\sigma, \tau\rangle$ is a polynomially growing subgroup of $\operatorname{Out}\left(F_{r}\right)$. By Bestvina, Feighn, and Handel's criterion for unipotence [6, Proposition 3.5], the group $\langle\sigma, \tau\rangle$ is a unipotent polynomially growing subgroup. Therefore, by the Kolchin theorem for $\operatorname{Out}\left(F_{r}\right)$ (see Theorem 3.2) there is a filtered graph $\emptyset=\Gamma_{0} \subsetneq \Gamma_{1} \subsetneq \cdots \Gamma_{k}=\Gamma$ with each step in the filtration a single edge, so that $\langle\sigma, \tau\rangle$ is realized as a group of upper-triangular homotopy equivalences of $\Gamma$ with respect to the filtration. Let $\hat{\sigma}$ and $\hat{\tau}$ be the realizations of the generators. Since $\sigma$ and $\tau$ are UPG, every $\hat{\sigma}$-periodic Nielsen path is $\hat{\sigma}$-Nielsen and every $\hat{\tau}$-periodic Nielsen path is $\hat{\tau}$-Nielsen.

Since $i(A, B)>0, \mathcal{C}(A, B)$ contains a rectangle, the contrapositive of Lemma 7.2 implies that either (up to relabeling) $\hat{\sigma}$ has a linear edge $E_{i}$ with suffix $u_{i}$ that grows linearly under $\hat{\tau}$ (as in Example 4.14 where, using the upper triangular representatives on the rose with edges $\bar{a}, \bar{b}, \bar{c}, \hat{\sigma}(\bar{a})=\bar{a} \bar{b}$, and $\bar{b}$ is $\hat{\tau}$-linear); or there is an edge $E_{i}$ so that the $\hat{\sigma}$ and $\hat{\tau}$ suffixes are powers of primitive Nielsen paths which generate non-equal cyclic subgroups and both suffixes are Nielsen for both automorphisms (as in Example 4.13 where, again using the representatives on the rose with edges $\bar{a}, \bar{b}, \bar{c}$, the $\hat{\sigma}$ and $\hat{\rho}$ suffixes of $\bar{a}$ are respectively $\bar{b}$ and $\bar{c}$ ). This gives two cases. In each case the proof generalizes the analysis of the appropriate guiding example.

Case 1. Let $E_{i}$ be the lowest edge in the filtration such that its suffix under one automorphism grows linearly under the other, and without loss of generality suppose that the $\hat{\sigma}$ suffix $u_{i}$ grows linearly under $\hat{\tau}$. We will use as a ping-pong set

$$
P=\left\{\left[\omega\left(E_{i}\right)\right] \mid \omega \in\langle\hat{\sigma}, \hat{\tau}\rangle\right\}
$$

the orbits of (the based homotopy class of) $E_{i}$ under tightening after applying elements of the group generated by $\hat{\sigma}$ and $\hat{\tau}$. Since a tight path is a unique representative of a based homotopy class the proof will focus on the tight representatives and the homotopy class will be suppressed. All of these classes have tight representatives of the form $E_{i} w$ with $w \subseteq \Gamma_{i-1}$ a tight path based at $t\left(E_{i}\right)$, since the group is upper triangular with respect to this filtration. Let

$$
P_{\sigma}=\left\{p \in P \mid \theta\left(\left[E_{i} u_{i}^{3}\right], p\right) \geq \theta\left(\left[E_{i} u_{i}^{3}\right],\left[E_{i} u_{i}^{2}\right]\right) \text { or } \theta\left(\left[E_{i} \bar{u}_{i}^{3}\right], p\right) \geq \theta\left(\left[E_{i} \bar{u}_{i}^{3}\right],\left[E_{i} \bar{u}_{i}^{2}\right]\right)\right\}
$$

and $P_{\tau}=P \backslash P_{\sigma}$ be a partition of $P$. It is clear that $P$ and $P_{\sigma}$ are non-empty, and we will show in the course of the proof that $P_{\tau}$ is non-empty. Let $\gamma_{k}$ be the common initial segment of $\left[u_{i}^{k}\right]$ and $\left[u_{i}^{k+1}\right]$, and $\gamma_{-k}$ the common initial segment of $\left[\bar{u}_{i}^{k}\right]$ and $\left[\bar{u}_{i}^{k+1}\right]$. Note that $\left[\bar{u}_{i}^{k} \gamma_{1}\right]=\gamma_{-(k-1)}$, the paths $\gamma_{j}$ are an increasing sequence of paths, and that $\left[\hat{\sigma}\left(\gamma_{j}\right)\right]=\gamma_{j} u^{\prime}$ where $u^{\prime}$ is the $\hat{\sigma}-$ Nielsen path associated to an exceptional $\hat{\sigma}$-Nielsen subpath of the primitive $\hat{\sigma}$-Nielsen path associated to $u_{i}$ if one exists.

We claim $\hat{\sigma}^{ \pm 3 N}\left(P_{\tau}\right) \subseteq P_{\sigma}$ for $N \neq 0$. The argument will be symmetric for negative powers, so suppose $N>0$. Consider $E_{i} w \in P_{\tau}$; we calculate

$$
\left[\hat{\sigma}^{3 N}\left(E_{i} w\right)\right]=E_{i}\left[\left[u_{i}^{3 N}\right] \hat{\sigma}^{3 N}(w)\right] .
$$

We must show that $E_{i} \gamma_{2}$ is the initial part of the path $\left[\hat{\sigma}^{3 N}\left(E_{i} w\right)\right]$. To establish this it suffices to show that $\left[\hat{\sigma}^{3 N}(w)\right]$ does not start with $\gamma_{-k}$ for some $k>3 N-1$. For a contradiction suppose $\left[\hat{\sigma}^{3 N}(w)\right]=\gamma_{-k} w^{\prime}$ for some $k>3 N-1$. Consider the $\hat{\sigma}$-canonical decomposition of $\gamma_{-k} w^{\prime}$. Either this agrees with the $\hat{\sigma}$-canonical decomposition of $\gamma_{-k}$, or the last edge of $\gamma_{-k}$ participates in a maximal exceptional subpath of $w^{\prime}$, so that the decomposition of $\gamma_{-k} w^{\prime}$ is obtained from $\gamma_{-(k-1)}$ and some $w^{\prime \prime}$. In either case, since every edge of $w$ is lower than the linear family associated to $u_{i},\left[\hat{\sigma}^{-3 N}\left(w^{\prime \prime}\right)\right]$ does not overlap $\left[u_{i}^{k}\right]$ in $\gamma_{k}$, and we have

$$
w=\left[\hat{\sigma}^{-3 N}\left(\gamma_{-(k-1)} w^{\prime \prime}\right)\right]=\gamma_{-(k-1)}\left[\hat{\sigma}^{-3 N}\left(w^{\prime \prime}\right)\right] .
$$


Since $k>3 N-1$, this implies $E_{i} w \in P_{\sigma}$, but we supposed $E_{i} w \notin P_{\sigma}$. Therefore, $E_{i}\left[u_{i}^{3 N} \hat{\sigma}^{3 N}(w)\right]$ has $E_{i} \gamma_{2}$ as an initial segment, so that $\hat{\sigma}^{3 N}\left(E_{i} w\right) \in P_{\sigma}$. The argument for negative powers is symmetric.

Next we claim $\hat{\tau}^{ \pm N}\left(P_{\sigma}\right) \subseteq P_{\tau}$ for $N \neq 0$. Let $v_{i}$ be the $\hat{\tau}$ suffix of $E_{i}$ (possibly trivial). Since $u_{i}$ grows linearly under $\tau, \gamma_{1}$ must contain a $\hat{\tau}$-linear edge or $\hat{\tau}$-linear exceptional path in its $\hat{\tau}$ decomposition. Neither $v_{i}$ nor $\bar{v}_{i}$, which are $\hat{\tau}$-Nielsen, can contain a $\hat{\tau}$-linear component in their $\hat{\tau}$-canonical decomposition as $v_{i}$ is a $\hat{\tau}$ suffix. A similar statement holds for $\gamma_{-1}$. Thus $v_{i}$ and $\bar{v}_{i}$ do not have $\gamma_{2}$ or $\gamma_{-2}$ as an initial segment. Consider the highest $\hat{\tau}$-linear edge of $\gamma_{1}$; since $\hat{\tau}$ is upper-triangular this edge cannot be canceled when tightening $\hat{\tau}^{N}\left(\gamma_{1}\right)$, so $\left[\hat{\tau}^{ \pm N}\left(\gamma_{2}\right)\right]$ has at most $\gamma_{1}$ in common with $\gamma_{2}$ (and similarly at most $\gamma_{-1}$ in common with $\gamma_{-2}$ ). Finally, suppose $E_{i} \gamma_{2} w \in P_{\sigma}$ is a tight representative. By the minimality in the choice of $E_{i}$, the highest $\hat{\tau}$-linear edge of $w$ is at most the same height as that in $\gamma_{2}$, so the highest $\hat{\tau}$-linear edges of $\gamma_{2} w$ do not cancel in the tightening of $\hat{\tau}^{ \pm N}\left(\gamma_{2}\right) \hat{\tau}^{ \pm N}(w)$. Putting this all together, the result $\left[\hat{\tau}^{ \pm N}\left(E_{i} \gamma_{2} w\right)\right]$ has at most $E_{i} \gamma_{1}$ in common with $E_{i}\left[u_{i}^{3}\right]$. Applying similar reasoning to $E_{i} \gamma_{-2} w^{\prime}$, we conclude $\hat{\tau}^{ \pm N}\left(P_{\sigma}\right) \subseteq P_{\tau}$ (this shows in particular that $P_{\tau}$ is non-empty). So by the ping-pong lemma $\left\langle\sigma^{3}, \tau^{3}\right\rangle \leq\left\langle\sigma^{3}, \tau\right\rangle \cong F_{2}$ as required.

Case 2. Suppose no $\hat{\sigma}$-suffix is $\hat{\tau}$-growing and vise-versa, and that there is an edge $E$ such that $\hat{\sigma}(E)=E u$ and $\hat{\tau}(E)=E v$, and the associated primitive Nielsen paths $u^{\prime}$ and $v^{\prime}$ do not generate isomorphic subgroups of $\pi_{1}(\Gamma, t(E))$. Since $v$ is not $\hat{\sigma}$-growing it is $\hat{\sigma}$-periodic, thus $[\hat{\sigma}(v)]=v$; similarly $[\hat{\tau}(u)]=u$. By hypothesis, $u_{*}, v_{*} \in \pi_{1}(\Gamma, t(E))$ generate a rank two free group $G$. Further, for $\omega \in\langle\hat{\sigma}, \hat{\tau}\rangle, \omega(E)=E w$ for some path $w$ so that $w_{*} \in\left\langle u_{*}, v_{*}\right\rangle$. It is immediate that $\omega \mapsto w_{*}$ is an isomorphism, hence $\langle\sigma, \tau\rangle \cong F_{2}$.

In either case $\left\langle\sigma^{3}, \tau^{3}\right\rangle \cong F_{2}$ as required.

Corollary 7.4. Suppose $\tilde{\sigma}, \tilde{\tau}$ are efficient Dehn twists satisfying every hypothesis of Lemma 7.3 except the condition on their image in $G L(r, \mathbb{Z} / 3 \mathbb{Z})$. Then $\left\langle\sigma^{9}, \tau^{9}\right\rangle \cong F_{2}$.

Proof. Since $\sigma$ and $\tau$ are Dehn twists, they are unipotent [11, and so $\sigma^{3}, \tau^{3}$ have trivial image in $G L(r, \mathbb{Z} / 3 \mathbb{Z})$. Therefore, by the lemma $\left\langle\left(\sigma^{3}\right)^{3},\left(\tau^{3}\right)^{3}\right\rangle \cong F_{2}$.

The culmination of this effort is a proof of a uniform McCarthy-type theorem for $\operatorname{Out}\left(F_{n}\right)$ in the linearly growing case.

Theorem 1.2. Suppose $\sigma$ and $\tau$ are linearly growing outer automorphisms of $F_{r}$. For $N=\left(48 r^{2}-48 r+3\right)|G L(r, \mathbb{Z} / 3 \mathbb{Z})|$ the subgroup $\left\langle\sigma^{N}, \tau^{N}\right\rangle$ is either abelian or free of rank two. Moreover, the latter case holds exactly when $i(A, B)>0$ for the Bass-Serre trees $A$ and $B$ of efficient representatives of Dehn-twist powers of $\sigma$ and $\tau$.

Proof. First, using train tracks Cohen and Lustig show that a unipotent linearly growing automorphism is a Dehn twist [11. Bestvina, Feighn, and Handel [6, Proposition 3.5] show that every polynomially growing outer automorphism with trivial image in $G L(r, \mathbb{Z} / 3 \mathbb{Z})$ is unipotent. Let $U=|G L(r, \mathbb{Z} / 3 \mathbb{Z})|$, so that $\sigma^{U}$ and $\tau^{U}$ are Dehn twists with trivial image in $G L(r, \mathbb{Z} / 3 \mathbb{Z})$ and efficient representatives on graphs of groups $\bar{A}$ and $\bar{B}$. If $i(A, B)>0$ then by either Lemma 7.1 or 7.3 since $N=48 r^{2}-48 r+3$ is divisible by 3 , the group $\left\langle\sigma^{U N}, \tau^{U N}\right\rangle \cong F_{2}$; otherwise by Lemma 4.1, $\sigma^{U}, \tau^{U}$ commute.

Acknowledgements. I thank Marc Culler for his guidance in the completion of my thesis. I am also grateful to my committee, Daniel Groves, Lee Mosher, Peter Shalen, and Kevin Whyte, for their careful reading of this work and helpful remarks. Samuel Taylor and David Futer's encouragement was most welcome in achieving the uniform power. The anonymous 
referee's thorough remarks improved the exposition, I very much appreciate their enthusiasm for examples. Finally, throughout writing my thesis I had great conversations with members of the geometric group theory community too numerous to list here.

\section{References}

[1] Steven C. Althoen, A geometrical realization of a construction of Bass and Serre, J. Pure Appl. Algebra 5 (1974), 233-237, DOI 10.1016/0022-4049(74)90034-6. MR0358754

[2] Jason Behrstock, Mladen Bestvina, and Matt Clay, Growth of intersection numbers for free group automorphisms, J. Topol. 3 (2010), no. 2, 280-310, DOI 10.1112/jtopol/jtq008. MR2651361

[3] Edgar A. Bering IV, Length Function Compatibility for Group Actions on Real Trees (2017), preprint, available at http://arxiv.org/abs/1708.07078v2.

[4] M. Bestvina, M. Feighn, and M. Handel, Laminations, trees, and irreducible automorphisms of free groups, Geom. Funct. Anal. 7 (1997), no. 2, 215-244, DOI 10.1007/PL00001618. MR1445386

[5] The Tits alternative for Out $\left(F_{n}\right)$. I. Dynamics of exponentially-growing automorphisms, Ann. of Math. (2) 151 (2000), no. 2, 517-623, DOI 10.2307/121043. MR1765705

[6] ㄴ The Tits alternative for Out $\left(F_{n}\right)$. II. A Kolchin type theorem, Ann. of Math. (2) 161 (2005), no. 1, 1-59, DOI 10.4007/annals.2005.161.1. MR2150382

[7] Mladen Bestvina and Mark Feighn, Outer Limits (1992), preprint.

[8] J. C. Chipman, van Kampen's theorem for n-stage covers, Trans. Amer. Math. Soc. 192 (1974), 357-370, DOI 10.2307/1996841. MR0339122

[9] Matt Clay and Alexandra Pettet, Twisting out fully irreducible automorphisms, Geom. Funct. Anal. 20 (2010), no. 3, 657-689, DOI 10.1007/s00039-010-0072-9. MR2720227

[10] Marshall M. Cohen and Martin Lustig, Very small group actions on R-trees and Dehn twist automorphisms, Topology 34 (1995), no. 3, 575-617, DOI 10.1016/0040-9383(94)00038-M. MR1341810

[11] (1999), no. 2, 179-200, DOI 10.1007/s000140050085. MR1691946

[12] Daryl Cooper, Automorphisms of free groups have finitely generated fixed point sets, J. Algebra 111 (1987), no. 2, 453-456, DOI 10.1016/0021-8693(87)90229-8. MR916179

[13] Marc Culler and John W. Morgan, Group actions on R-trees, Proc. London Math. Soc. (3) 55 (1987), no. 3, 571-604, DOI 10.1112/plms/s3-55.3.571. MR907233

[14] Koji Fujiwara, Subgroups generated by two pseudo-Anosov elements in a mapping class group. I. Uniform exponential growth, Groups of diffeomorphisms, Adv. Stud. Pure Math., vol. 52, Math. Soc. Japan, Tokyo, 2008, pp. 283-296. MR2509713

[15] Pritam Ghosh, Applications of weak attraction theory in $\operatorname{Out}\left(F_{n}\right)$ (2013), preprint, available at https://arxiv.org/abs/1306.6049

[16] Vincent Guirardel, Cœur et nombre d'intersection pour les actions de groupes sur les arbres, Ann. Sci. École Norm. Sup. (4) 38 (2005), no. 6, 847-888, DOI 10.1016/j.ansens.2005.11.001 (French, with English and French summaries). MR2216833

[17] Funda Gültepe, Fully irreducible automorphisms of the free group via Dehn twisting in $\sharp_{k}\left(S^{2} \times S^{1}\right)$, Algebr. Geom. Topol. 17 (2017), no. 3, 1375-1405, DOI 10.2140/agt.2017.17.1375. MR3677931

[18] Hessam Hamidi-Tehrani, Algorithms in the surface mapping class groups, Columbia University, 1997. Thesis (Ph.D.) MR2695693

[19] P. J. Higgins, The fundamental groupoid of a graph of groups, J. London Math. Soc. (2) 13 (1976), no. 1, 145-149, DOI 10.1112/jlms/s2-13.1.145. MR0401927

[20] N. V. Ivanov, Algebraic properties of the Teichmüller modular group, Dokl. Akad. Nauk SSSR 275 (1984), no. 4, 786-789 (Russian). MR745513

[21] Ilya Kapovich and Martin Lustig, Geometric intersection number and analogues of the curve complex for free groups, Geom. Topol. 13 (2009), no. 3, 1805-1833, DOI 10.2140/gt.2009.13.1805. MR2496058

[22] - Ping-pong and outer space, J. Topol. Anal. 2 (2010), no. 2, 173-201, DOI 10.1142/S1793525310000318. MR2652906

[23] E. R. Kolchin, Algebraic matric groups and the Picard-Vessiot theory of homogeneous linear ordinary differential equations, Ann. of Math. (2) 49 (1948), 1-42, DOI 10.2307/1969111. MR0024884

[24] Johanna Mangahas, Uniform uniform exponential growth of subgroups of the mapping class group, Geom. Funct. Anal. 19 (2010), no. 5, 1468-1480, DOI 10.1007/s00039-009-0038-y. MR2585580

[25] Dan Margalit and Saul Schleimer, Dehn twists have roots, Geom. Topol. 13 (2009), no. 3, 1495-1497, DOI 10.2140/gt.2009.13.1495. MR2496051

[26] John McCarthy, A "Tits-alternative" for subgroups of surface mapping class groups, Trans. Amer. Math. Soc. 291 (1985), no. 2, 583-612, DOI 10.2307/2000100. MR800253

[27] Frédéric Paulin, Sur les automorphismes de groupes libres et de groupes de surface, Astérisque 339 (2011), Exp. No. 1023, ix, 323-356 (French, with French summary). Séminaire Bourbaki. Vol. 2009/2010. Exposés 1012-1026. MR2906359

[28] Peter Scott and Terry Wall, Topological methods in group theory, Homological group theory (Proc. Sympos., Durham, 1977), London Math. Soc. Lecture Note Ser., vol. 36, Cambridge Univ. Press, Cambridge-New York, 1979, pp. 137-203. MR564422 
[29] Jean-Pierre Serre, Trees, Springer Monographs in Mathematics, Springer-Verlag, Berlin, 2003. Translated from the French original by John Stillwell; Corrected 2nd printing of the 1980 English translation. MR1954121

[30] John R. Stallings, Topology of finite graphs, Invent. Math. 71 (1983), no. 3, 551-565, DOI 10.1007/BF02095993. MR695906

[31] Samuel J. Taylor, Right-angled Artin groups and $\operatorname{Out}\left(\mathbb{F}_{n}\right)$ I. Quasi-isometric embeddings, Groups Geom. Dyn. 9 (2015), no. 1, 275-316, DOI 10.4171/GGD/313. MR3343354

[32] Marvin D. Tretkoff, A topological approach to the theory of groups acting on trees, J. Pure Appl. Algebra 16 (1980), no. 3, 323-333, DOI 10.1016/0022-4049(80)90037-7. MR558496

Edgar A. Bering IV

Department of Mathematics, Wachman

Hall, Temple University, 1805 Broad

Street, Philadelphia, PA 19122

U.S. A.

edgar.bering@temple.edu 